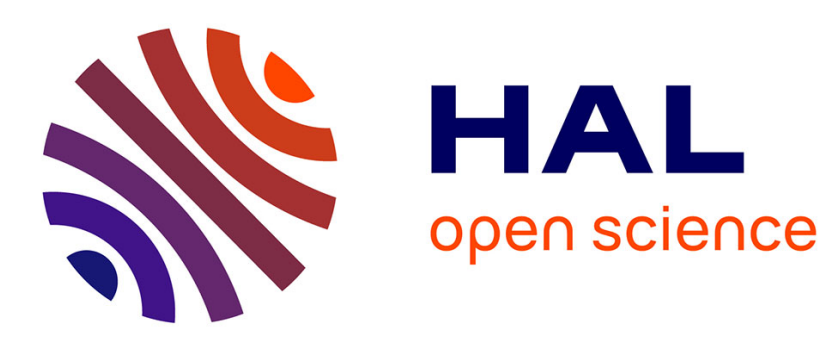

\title{
Multi-frequency model reduction for uncertainty quantification in computational vibroacoutics
}

Justin Reyes, Christophe Desceliers, Christian Soize, Laurent Gagliardini

\section{To cite this version:}

Justin Reyes, Christophe Desceliers, Christian Soize, Laurent Gagliardini. Multi-frequency model reduction for uncertainty quantification in computational vibroacoutics. Computational Mechanics, 2022, 69, pp.661-682. 10.1007/s00466-021-02109-y . hal-03381359

\section{HAL Id: hal-03381359 \\ https://hal.science/hal-03381359}

Submitted on 3 Jan 2022

HAL is a multi-disciplinary open access archive for the deposit and dissemination of scientific research documents, whether they are published or not. The documents may come from teaching and research institutions in France or abroad, or from public or private research centers.
L'archive ouverte pluridisciplinaire HAL, est destinée au dépôt et à la diffusion de documents scientifiques de niveau recherche, publiés ou non, émanant des établissements d'enseignement et de recherche français ou étrangers, des laboratoires publics ou privés. 


\title{
Multi-frequency model reduction for uncertainty quantification in computational vibroacoutics
}

\author{
Justin Reyes $^{\mathrm{a}}$, Christophe Desceliers ${ }^{*}$, , Christian Soize ${ }^{\mathrm{a}}$, Laurent Gagliardini ${ }^{\mathrm{b}}$ \\ ${ }^{a}$ Univ Gustave Eiffel, MSME UMR 8208, 5 bd Descartes, 77454 Marne-La-Vallée, France \\ ${ }^{b}$ PSA Groupe, Direction Technique et Industrielle, Centre Technique de Vélizy A, Route de Gisy, 78140 Vélizy \\ Villacoublay, France
}

\begin{abstract}
This work is devoted to the vibroacoustics of complex systems over a broad-frequency band of analysis. The considered system is composed of a complex structure coupled with an internal acoustic cavity. On one hand, the global displacements are associated with the main stiff part and on the other hand, the local displacements are associated with the preponderant vibrations of the flexible subparts. Such complex structures induce interweaving of these two types of displacements, which introduce an overlap of the usual three frequency bands (low-, medium- and high-frequency bands (LF, MF, and HF). A reduced-order computational vibroacoustic model is constructed by using a classical modal analysis with the elastic and acoustic modes. Nevertheless, the dimension of such reduced-order model (ROM) is still important when there is an overlap for each one of the three frequency bands. A multi-frequency reduced-order model is then constructed for the structure over the LF, MF, and HF bands. The strategy is based on a multilevel projection consisting in introducing three reduced-order bases that are obtained by using a spatial filtering methodology. To filter out the local displacements in the structure, a set of global shape functions is introduced. In addition, a classical ROM using acoustic modes is carried out for the acoustic cavity. Then, the coupling between the multilevel ROM and the acoustic ROM is presented. A nonparametric probabilistic modeling is then proposed to take into account the model uncertainties induced by modeling errors that increase with the frequency. The proposed approach is applied to a large-scale computational vibroacoustic model of a car.
\end{abstract}

Key words: Vibroacoustics, Reduced-order model, Multilevel, Broad-frequency band, Uncertainty Quantification, Automobile

\section{Introduction}

In this work, the dynamical analysis of complex vibroacoustic systems is developed in a broad-frequency band. The complex system can be separated in two parts, a complex structure (such as the structure of a car) coupled with an internal acoustic cavity (such as the cockpit of

\footnotetext{
*Corresponding author: Christophe Desceliers, christophe.desceliers@univ-eiffel.fr

Email addresses: justin.reyes@univ-eiffel.fr (Justin Reyes), christophe.desceliers@univ-eiffel.fr(Christophe Desceliers), christian.soize@univ-eiffel.fr (Christian Soize), laurent .gagliardini@mpsa.com (Laurent Gagliardini)

Preprint of the published paper in Computational Machanics, 2021.
} 
the car). The complex structure is defined by a complex geometry, made up of heterogeneous materials and more specifically, characterized by the presence of numerous structural levels. The structure is made up of a stiff main part embedding various flexible sub-parts. In addition to the usual global displacement elastic modes linked with the stiff skeleton, there are a numerous local elastic modes that are associated with the predominant vibrations of the flexible subparts. Such complex structures can be found, for instance in aeronautics and aerospace, but above all in the automotive industry [1, 2, 3, 4]. There are two main difficulties caused by the presence of local displacements in the structure. Firstly, the modal density may increase abruptly from low frequencies, causing a high dimensional reduced-order model (ROM) within modal analysis. Secondly, such a ROM may not be robust enough with respect to uncertainties caused by the presence of the numerous local displacements, which are known to be very sensitive to uncertainties. The engineering objectives for such complex structures are to obtain a computational model with a robust prediction for the global displacements at observation points that belong to the main stiff part.

For a vibroacoustic system composed of a complex elastic structure coupled with an internal acoustic cavity, characterized by the presence of numerous local elastic modes entangled with global elastic modes, the research are not plentiful. Nonetheless for the structural part, numerous works have been published into the literature. In the experimental modal analysis framework, a spatial filtering method of the local displacements [5], based on regularization schemes, has been proposed in [6]. For computational models, the Guyan condensation technique [7] has also been used, consisting in introducing master structural nodes in which the mass matrix is condensed. It allows the filtering of local displacements to be obtained. The downside is the complexity of the choice of the master nodes [8]. Filtering using the lumped mass matrix approximations has also been introduced by [9, 10, 11] but it depends on the mesh and cannot easily be adjusted. The construction [12] of a global displacement basis using a coarse mesh can yield important errors for the elastic energy. Other methods for extracting the long-wavelength elastic modes of the main structure, like the interface substructuring, have also been proposed [13]. Moreover, computational approaches based on the use of image processing [14] have been proposed for identifying the global elastic modes, in which the global displacements are considered as the eigenvectors of the frequency mobility matrix [15]. The extrapolation of the dynamical response using a sparse representation constructed with a few elastic modes has also been proposed in [16]. In the low-frequency (LF) band, for slender structures exhibiting a high modal density, simplified equivalent models have been proposed in [17, 18] and homogenization has been suggested in [19]. Though, using these approaches, the simplification of the model is not automatic and generally requires an expertise and a validation.

For complex structures for which the elastic modes are neither purely global nor purely local displacements, the increasing of the dimension of the ROM based on the classical modal analysis proves to be inconvenient. The methodology to sort the elastic modes depending to whether they are global displacements or local displacements is also irrelevant because the elastic modes are an association of both types of displacement. It is well known that large amplitudes of the local displacements are difficult to distinguish from the global ones using the modal shapes. Such a difficulty increases with the frequency.

Generally, in the case of a complex structure, an elastic mode is constituted of global displacements (long wavelength deformations) assorted with local displacements (short wavelength deformations) of differentiated structural level. One important observation is that as the frequency increases, the global displacements in the elastic modes are less and less perceptible. The fact is that they are covered by high-amplitude local displacements. 
Nevertheless, substructuring techniques have been used for trying to solve this separation problem. The substructuring techniques have deeply been studied [20, 21, 22]. As written in [23]: "historically, the concept of substructures was first introduced by Argyris and Kelsey in 1959 [24] and by Przemieniecki in 1963 [25] and was extended by Guyan and Irons [7, 26]. Hurty [27, 28] considered the case of two substructures coupled through a geometrical interface. Finally, Craig and Bampton [29] have adapted the Hurty method. Many variants have been proposed for improving substructuring techniques [30, 31, 32, 33, 34], notably for the complex dynamical systems with many auxiliaries considered as substructures from Benfield and Hruda [35]. A new group of methods has been introduced for two coupled subtructures with fixed geometrical interface using structural modes with free geometry interface, as proposed by Craig and Bampton, MacNeal [36], and Rubin [37]. Then the Lagrange multipliers have been applied to write the coupling on the geometrical interface [38, 39, 40, 41]". The substructuring needs discarding the component modes associated with flexible subparts, to eliminate their associated local displacements. In the proposed approach, there is no clear boundary between the skeleton and the substructures, that is to say, between the main stiff part and the flexible subparts. This property can be explained by the complex geometry of the structure that is constructed in order to have a continuous series of structural levels, instead of a clear separation, in addition to the various embedded equipments. In this type of setup, the notion of local displacements is relative. It is worth mentioning that, in comparison to the usual global displacements that are present in the LF band, the local displacements associated with the structural sub-levels (which can also appear in the LF band) are characterized by a high-complexity level, similarly to those in the $\mathrm{HF}$ band. Therefore, for the complex structure studied, there is an overlap of the three vibration regimes (LF, MF, and HF bands).

Regarding the uncertainties in the computational model, the probabilistic framework is well suited to construct the stochastic models and to solve the inverse problems for the identification of the probabilistic models of uncertainties. Thereafter, we present the framework limited to the probabilistic approaches for uncertainty quantification. To take into account model-parameter uncertainties, model uncertainties induced by modeling errors, and variabilities in the real dynamical system, different probabilistic approaches can be used (see [42]).

The parametric probabilistic approach is well adapted to model-parameter uncertainties, at least for a sufficient small number of parameters. It involves the construction of prior and posterior stochastic models of uncertain model parameters linked, for instance, to materials properties, to geometry, to boundary conditions, etc. Concerning such a parametric probabilistic approach of uncertainties, the reader is referred, for example, to [43, 44, 45, 46, 47] for random vibration and structural dynamics, to [48, 49, 50, 51] for stochastic computational mechanics, to [52, 53, 54] for random fields and polynomial chaos expansions, and to [55, 42] for a general overview on uncertainty quantification. This approach is computationally efficient for both the computational model and its resulting ROM [56, 57], and for large-scale statistical inverse problems [58, 59, 60, 61, 62, 63]. The main limit of this approach is that it does not take into account neither the model uncertainties induced by modeling errors introduced during the construction of the computational model nor the uncertainties caused by the use of a ROM.

The nonparametric probabilistic approach [64, 42] allows model uncertainties induced by modeling errors to be taken into account linear dynamical systems. The modus operandi is done in two stages. Firstly, the construction of a linear ROM of dimension $n$ using the linear computational model with $m$ degrees of freedom (DOFs) and a reduced-order basis (ROB) of dimension $n$. Then, a linear stochastic ROM is built by replacing the deterministic matrices of the linear ROM by random matrices for which the probability distributions are constructed [42] using the 
Maximum Entropy (MaxEnt) Principle [65, 66] from Information Theory. The construction of the linear stochastic ROM is fulfilled under the constraint generated from the available information such as algebraic positiveness, integrability of the inverse, and some statistical information. This approach enlarged for different family of random matrices and for linear boundary value problems [67, 68, 42]. For linear problems, experimental validations and applications to statistical inverse problems can be found in [69] for composites, [70] for viscoelasticity, [71, 72, 73] for dynamic substructuring, [74, 1] for vibroacoustics, [75] for robust design and optimization, and [76] for the identification and sampling the Bayesian posteriors of high-dimensional symmetric positive-definite random matrices for data-driven updating of computational models. An extension to the nonlinear geometrical effects in structural analysis has also been proposed [77, 78, 79].

Objectives and novelty of the paper. Recently, a method has been proposed in [80, 81] to separate the local displacements and the global displacements using a spatial filtering (on wavelength). Moreover, the multilevel stochastic approach for structural dynamics has been introduced in [81] to adapt the level of uncertainties in each frequency band: LF, MF, and HF. This work presents an extension to the case of vibroacoustic systems based on a reformulation of the construction of the multilevel ROM for the structural part. An objective of this work is to propose a predictive stochastic multilevel ROM whose dimension is inferior to the usual ROM constructed by using the classical modal analysis. Another very important objective is that the algorithms developed can be used for very large computational models without encountering problems related to the limitation of RAM and with numerical costs that remain low. This last objective requires to develop a methodology and algorithms, which are not intrusive with respect to commercial software.

Organization of the paper. Section2 2 is devoted to the construction of the classical reduced-order computational vibroacoustic model that is carried out by projecting the full-order computational vibroacoustic model on the elastic and acoustic modes. Section 3 presents the principle of the spatial filtering. Section 4 is devoted to the construction of a multilevel basis and the associated algorithms are detailed. Moreover, a numerical application of the multilevel reduced-order computational model is presented. Finally in Section 5, the construction of a stochastic multilevel reduced-order computational model in vibroacoustics is presented. The probabilistic model of random matrices is constructed in the framework of the nonparametric probabilistic approach of model uncertainties. The numerical application is devoted to a large computational vibroacoustic model of an automobile.

\section{Classical reduced-order model of a vibroacoustic computational model}

In this section, the construction of the reduced-order computational vibroacoustic model (ROM) is introduced for a vibroacoustic system (the car and the air in its cockpit). The ROM will be constructed by projecting the full-order computational vibroacoustic model on the elastic and acoustic modes. The solution is therefore computed with the well known modal analysis method.

\subsection{Full-order computational vibroacoustic model}

The vibroacoustic analysis is carried out over a broad-frequency band denoted by $\mathbb{B}$. The computational vibroacoustic model is built using the finite element method. The angular frequency $\omega$ belongs to the frequency band of analysis $\mathbb{B}=\left[\omega_{\min }, \omega_{\max }\right]$. Let $u(\omega)($ resp. $\mathbb{p}(\omega))$ 
be the vector of all the degrees of freedom corresponding to the nodal values on the finite element mesh of domain $\Omega_{\mathrm{s}}$ (resp. $\Omega_{\mathrm{f}}$ ) for displacement field $\mathbf{u}(\cdot, \omega)$ (resp. acoustic pressure field $p(\cdot, \omega))$. Let $n_{\text {dof }}^{\mathrm{s}}\left(\right.$ resp. $\left.n_{\text {dof }}^{\mathrm{f}}\right)$ be the dimension of vector $u(\omega)($ res. $\mathbb{p}(\omega))$. The full-order computational vibroacoustic model is written as

$$
\begin{aligned}
& \left(-\omega^{2}\left[\mathbb{M}^{\mathrm{s}}\right]+i \omega\left[\mathbb{D}^{\mathrm{s}}\right]+\left[\mathbb{K}^{\mathrm{s}}\right]\right) u(\omega)+[\mathbb{C}] \mathbb{p}(\omega)=\mathbb{F}^{\mathrm{s}}(\omega), \\
& \left(-\omega^{2}\left[\mathbb{M}^{\mathrm{f}}\right]+i \omega\left[\mathbb{D}^{\mathrm{f}}\right]+\left[\mathbb{K}^{\mathrm{f}}\right]\right) \mathbb{p}(\omega)+\omega^{2}[\mathbb{C}]^{T} u(\omega)=\mathbf{0},
\end{aligned}
$$

in which $\left[\mathbb{M}^{\mathrm{s}}\right],\left[\mathbb{D}^{\mathrm{s}}\right]$, and $\left[\mathbb{K}^{\mathrm{s}}\right]$ are the $n_{\mathrm{dof}}^{\mathrm{s}} \times n_{\text {dof }}^{\mathrm{s}}$ positive-definite symmetric real mass, damping, and stiffness matrices for the structure, where $\left[\mathbb{M}^{\mathrm{f}}\right]\left(\right.$ resp. $\left[\mathbb{D}^{\mathrm{f}}\right]$ and $\left.\left[\mathbb{K}^{\mathrm{f}}\right]\right)$ is the $n_{\text {dof }}^{\mathrm{f}} \times n_{\text {dof }}^{\mathrm{f}}$ positivedefinite (resp. positive) symmetric real mass (resp. damping and stiffness) matrix for the acoustic cavity, where $[\mathbb{C}]$ is the $n_{\text {dof }}^{\mathrm{s}} \times n_{\text {dof }}^{\mathrm{f}}$ coupling matrix and where $\mathbb{F}^{\mathrm{s}}$ is the finite-element vector of the external forces.

\subsection{Elastic modes}

Let $0<\lambda_{1}^{\mathrm{s}} \leq \ldots \leq \lambda_{n^{\mathrm{s}}}^{\mathrm{s}}$ be the $n^{\mathrm{s}} \ll n_{\mathrm{dof}}^{\mathrm{s}}$ smallest eigenvalues such that

$$
\left[\mathbb{K}^{\mathrm{s}}\right] \varphi_{\alpha}^{\mathrm{s}}=\lambda_{\alpha}^{\mathrm{s}}\left[\mathbb{M}^{\mathrm{s}}\right] \varphi_{\alpha}^{\mathrm{s}} .
$$

The eigenvectors $\varphi_{1}^{\mathrm{s}}, \ldots, \varphi_{n^{\mathrm{s}}}^{\mathrm{s}}$ in $\mathbb{R}_{\text {dof }}^{n^{\mathrm{s}}}$ associated with the eigenvalues verify the usual orthogonality properties and normalization,

$$
\left(\varphi_{\beta}^{\mathrm{s}}\right)^{T}\left[\mathbb{M}^{\mathrm{s}}\right] \varphi_{\alpha}^{\mathrm{s}}=\delta_{\alpha \beta},\left(\varphi_{\beta}^{\mathrm{s}}\right)^{T}\left[\mathbb{K}^{\mathrm{s}}\right] \varphi_{\alpha}^{\mathrm{s}}=\lambda_{\alpha}^{\mathrm{s}} \delta_{\alpha \beta} .
$$

The LF, MF, and HF bands are $\left[0, \omega_{\mathrm{L}}\right],\left[\omega_{\mathrm{L}}, \omega_{\mathrm{M}}\right]$, and $\left[\omega_{\mathrm{M}}, \omega_{\mathrm{H}}\right]$ (in rad.s $\left.{ }^{-1}\right)$ where the three bounds $\omega_{\mathrm{L}}, \omega_{\mathrm{M}}$, and $\omega_{\mathrm{L}}$ are assumed to be already known. Furthermore, let $n_{\mathrm{L}}, n_{\mathrm{M}}$, and $n_{\mathrm{H}}$ be the number of structural elastic modes in the LF, MF, and HF bands, and let $n_{\mathrm{LM}}=n_{\mathrm{L}}+n_{\mathrm{M}}$. For the whole three LF, MF, and HF bands, let $\left[\Phi^{\mathrm{s}}\right]=\left[\varphi_{1}^{\mathrm{s}} \ldots \boldsymbol{\varphi}_{n^{\mathrm{s}}}^{\mathrm{s}}\right]$ be the $n_{\mathrm{dof}}^{\mathrm{s}} \times n^{\mathrm{s}}$ modal matrix. Let $\left[\Phi_{\mathrm{L}}^{\mathrm{s}}\right],\left[\Phi_{\mathrm{M}}^{\mathrm{s}}\right]$, and $\left[\Phi_{\mathrm{H}}^{\mathrm{s}}\right]$ be the matrices with dimensions $n_{\mathrm{dof}}^{\mathrm{s}} \times n_{\mathrm{L}}, n_{\mathrm{dof}}^{\mathrm{s}} \times n_{\mathrm{M}}$, and $n_{\mathrm{dof}}^{\mathrm{s}} \times n_{\mathrm{H}}$ whose columns are the elastic modes belonging to LF, MF, and HF bands respectively and such that

$$
\left[\Phi^{\mathrm{s}}\right]=\left[\left[\Phi_{\mathrm{L}}^{\mathrm{s}}\right]\left[\Phi_{\mathrm{M}}^{\mathrm{s}}\right]\left[\Phi_{\mathrm{H}}^{\mathrm{s}}\right]\right] .
$$

We also introduce the matrix $\left[\Phi_{\mathrm{LM}}^{\mathrm{s}}\right]$ that is defined by

$$
\left[\Phi_{\mathrm{LM}}^{\mathrm{s}}\right]=\left[\left[\Phi_{\mathrm{L}}^{\mathrm{s}}\right]\left[\Phi_{\mathrm{M}}^{\mathrm{s}}\right]\right] .
$$

\subsection{Acoustic modes}

Let $0=\lambda_{0}^{\mathrm{f}}<\lambda_{1}^{\mathrm{f}} \leq \ldots \leq \lambda_{n^{\mathrm{f}}}^{\mathrm{f}}$ be the $n^{\mathrm{f}}+1 \ll n_{\text {dof }}^{\mathrm{f}}$ smallest eigenvalues such that

$$
\left[\mathbb{K}^{\mathrm{f}}\right] \varphi_{\alpha}^{\mathrm{f}}=\lambda_{\alpha}^{\mathrm{f}}\left[\mathbb{M}^{\mathrm{f}}\right] \varphi_{\alpha}^{\mathrm{f}} .
$$

It should be noted that there is a zero eigenvalue $\lambda_{0}^{\mathrm{f}}$ because the dimension of the null space of matrix $\left[\mathbb{K}^{\mathrm{f}}\right]$ is 1 . The eigenvectors $\boldsymbol{\varphi}_{0}^{\mathrm{f}}, \boldsymbol{\varphi}_{1}^{\mathrm{f}}, \ldots, \boldsymbol{\varphi}_{n^{\mathrm{f}}}^{\mathrm{f}}$ in $\mathbb{R}_{\text {dof }}^{n_{\mathrm{f}}}$ associated with the eigenvalues verify the usual orthogonality properties and normalization, for all $\alpha$ and $\beta$ in $\left\{1, \ldots, n^{\mathrm{f}}\right\}$,

$$
\left(\varphi_{\beta}^{\mathrm{f}}\right)^{T}\left[\mathbb{M}^{\mathrm{f}}\right] \varphi_{\alpha}^{\mathrm{f}}=\delta_{\alpha \beta}, \quad\left(\varphi_{\beta}^{\mathrm{f}}\right)^{T}\left[\mathbb{K}^{\mathrm{f}}\right] \varphi_{\alpha}^{\mathrm{f}}=\lambda_{\alpha}^{\mathrm{f}} \delta_{\alpha \beta} .
$$


For the LF, MF, and HF bands, the $n_{\text {dof }}^{\mathrm{f}} \times n^{\mathrm{f}}$ modal matrix is defined as $\left[\Phi^{\mathrm{f}}\right]=\left[\varphi_{1}^{\mathrm{f}} \ldots \varphi_{n^{\mathrm{f}}}^{\mathrm{f}}\right]$ in which the eigenvector $\varphi_{0}^{\mathrm{f}}$, which is associated with the null eigenvalue $\lambda_{0}^{\mathrm{f}}$, has been omitted, assuming an almost closed (nonsealed wall) acoustic cavity [74, 82].

\subsection{Reduced-order computational vibroacoustic models for the LF-MF-HF and LF-MF bands}

In the LF, MF, and HF bands, the ROM is constructed by using the truncated modal expansion of vectors $u(\omega)$ and $p(\omega)$ such that

$$
u(\omega)=\left[\Phi^{\mathrm{s}}\right] \mathbf{q}^{\mathrm{s}}, \quad \mathbb{P}(\omega)=\left[\Phi^{\mathrm{f}}\right] \mathbf{q}^{\mathrm{f}} .
$$

Using these modal expansions in Eqs. (1) and 22 yield

$$
\begin{aligned}
& \left(-\omega^{2}\left[\mathcal{M}^{\mathrm{s}}\right]+i \omega\left[\mathcal{D}^{\mathrm{s}}\right]+\left[\mathcal{K}^{\mathrm{s}}\right]\right) \mathbf{q}^{\mathrm{s}}(\omega)+[\mathcal{C}] \mathbf{q}^{\mathrm{f}}(\omega)=\mathbf{f}^{\mathrm{s}}(\omega), \\
& \left(-\omega^{2}\left[\mathcal{M}^{\mathrm{f}}\right]+i \omega\left[\mathcal{D}^{\mathrm{f}}\right]+\left[\mathcal{K}^{\mathrm{f}}\right]\right) \mathbf{q}^{\mathrm{f}}(\omega)+\omega^{2}[\mathcal{C}]^{T} \mathbf{q}^{\mathrm{s}}(\omega)=\mathbf{0},
\end{aligned}
$$

in which $\left[\mathcal{M}^{\mathrm{s}}\right]=\left[\Phi^{\mathrm{s}}\right]^{T}\left[\mathbb{M}^{\mathrm{s}}\right]\left[\Phi^{\mathrm{s}}\right],\left[\mathcal{D}^{\mathrm{s}}\right]=\left[\Phi^{\mathrm{s}}\right]^{T}\left[\mathbb{D}^{\mathrm{s}}\right]\left[\Phi^{\mathrm{s}}\right]$, and $\left[\mathcal{K}^{\mathrm{s}}\right]=\left[\Phi^{\mathrm{s}}\right]^{T}\left[\mathbb{K}^{\mathrm{s}}\right]\left[\Phi^{\mathrm{s}}\right]$ are $n^{\mathrm{s}} \times n^{\mathrm{s}}$ positive-definite matrices, where $\left[\mathcal{M}^{\mathrm{f}}\right]=\left[\Phi^{\mathrm{f}}\right]^{T}\left[\mathbb{M}^{\mathrm{f}}\right]\left[\Phi^{\mathrm{f}}\right],\left[\mathcal{D}^{\mathrm{f}}\right]=\left[\Phi^{\mathrm{f}}\right]^{T}\left[\mathbb{D}^{\mathrm{f}}\right]\left[\Phi^{\mathrm{f}}\right]$, and $\left[\mathcal{K}^{\mathrm{f}}\right]=$ $\left[\Phi^{\mathrm{f}}\right]^{T}\left[\mathbb{K}^{\mathrm{f}}\right]\left[\Phi^{\mathrm{f}}\right]$ are $n^{\mathrm{f}} \times n^{\mathrm{f}}$ positive-definite matrices, where $[\mathcal{C}]=\left[\Phi^{\mathrm{s}}\right]^{T}[\mathbb{C}]\left[\Phi^{\mathrm{f}}\right]$, and where $\mathbf{f}^{\mathrm{s}}(\omega)=\left[\Phi^{\mathrm{s}}\right]^{T} \mathbb{F}^{\mathrm{s}}(\omega)$. Note that the orthogonality properties and normalization defined by Eqs. (3) and (4) yield $\left[\mathcal{M}^{\mathrm{s}}\right]=\left[I_{n^{\mathrm{s}}}\right]$ and $\left[\mathcal{K}^{\mathrm{s}}\right]=\left[\Lambda^{\mathrm{s}}\right]$ for the structure, and $\left[\mathcal{M}^{\mathrm{f}}\right]=\left[I_{n^{\mathrm{f}}}\right]$ and $\left[\mathcal{K}^{\mathrm{s}}\right]=\left[\Lambda^{\mathrm{f}}\right]$ for the cavity in which $\left[\Lambda^{\mathrm{s}}\right]_{\alpha \beta}=\lambda_{\alpha}^{\mathrm{s}} \delta_{\alpha \beta}$ and $\left[\Lambda^{\mathrm{f}}\right]_{\alpha \beta}=\lambda_{\alpha}^{\mathrm{f}} \delta_{\alpha \beta}$ are diagonal $n^{\mathrm{s}} \times n^{\mathrm{s}}$ and $n^{\mathrm{f}} \times n^{\mathrm{f}}$ matrices. Nevertheless, it is well known that such a ROM is not robust in the HF domain. In addition, this work focus on the LF and MF domains while the only purpose of computing the eigenvectors associated with eigenfrequencies into HF consists in carrying out an analysis for defining the spatial complexity level of the HF domains. Consequently, when the analysis is carried out into the LF and MF domains, the ROM defined by Eqs. (5) and (6) is not used. Instead, a truncated modal expansion of vectors $u(\omega)$ and $p(\omega)$ that are rather written as

$$
u(\omega)=\left[\Phi_{\mathrm{LM}}^{\mathrm{s}}\right] \mathbf{q}_{\mathrm{LM}}^{\mathrm{s}}, \quad \mathbb{P}(\omega)=\left[\Phi^{\mathrm{f}}\right] \mathbf{q}^{\mathrm{f}}
$$

Using such truncated modal expansions for projecting Eqs. (1) and (2) yields

$$
\begin{gathered}
\left(-\omega^{2}\left[\mathcal{M}_{\mathrm{LM}}^{\mathrm{s}}\right]+i \omega\left[\mathcal{D}_{\mathrm{LM}}^{\mathrm{s}}\right]+\left[\mathcal{K}_{\mathrm{LM}}^{\mathrm{s}}\right]\right) \mathbf{q}_{\mathrm{LM}}^{\mathrm{s}}(\omega)+\left[\mathcal{C}_{\mathrm{LM}}\right] \mathbf{q}^{\mathrm{f}}(\omega)=\mathbf{f}_{\mathrm{LM}}^{\mathrm{s}}(\omega), \\
\left(-\omega^{2}\left[\mathcal{M}^{\mathrm{f}}\right]+i \omega\left[\mathcal{D}^{\mathrm{f}}\right]+\left[\mathcal{K}^{\mathrm{f}}\right]\right) \mathbf{q}^{\mathrm{f}}(\omega)+\omega^{2}\left[\mathcal{C}_{\mathrm{LM}}\right]^{T} \mathbf{q}_{\mathrm{LM}}^{\mathrm{s}}(\omega)=\mathbf{0},
\end{gathered}
$$

in which $\left[\mathcal{M}_{\mathrm{LM}}^{\mathrm{s}}\right]=\left[\Phi_{\mathrm{LM}}^{\mathrm{s}}\right]^{T}\left[\mathbb{M}^{\mathrm{s}}\right]\left[\Phi_{\mathrm{LM}}^{\mathrm{s}}\right],\left[\mathcal{D}_{\mathrm{LM}}^{\mathrm{s}}\right]=\left[\Phi_{\mathrm{LM}}^{\mathrm{s}}\right]^{T}\left[\mathbb{D}^{\mathrm{s}}\right]\left[\Phi_{\mathrm{LM}}^{\mathrm{s}}\right]$, and $\left[\mathcal{K}_{\mathrm{LM}}^{\mathrm{s}}\right]=\left[\Phi_{\mathrm{LM}}^{\mathrm{s}}\right]^{T}\left[\mathbb{K}^{\mathrm{s}}\right]$ $\left[\Phi_{\mathrm{LM}}^{\mathrm{s}}\right]$ are $n_{\mathrm{LM}}^{\mathrm{s}} \times n_{\mathrm{LM}}^{\mathrm{s}}$ positive-definite matrices, where $\left[\mathcal{C}_{\mathrm{LM}}\right]=\left[\Phi_{\mathrm{LM}}^{\mathrm{s}}\right]^{T}[\mathbb{C}]\left[\Phi_{\mathrm{LM}}^{\mathrm{f}}\right]$, and where $\mathbf{f}_{\mathrm{LM}}^{\mathrm{s}}(\omega)=\left[\Phi_{\mathrm{LM}}^{\mathrm{s}}\right]^{T} \mathbb{F}^{\mathrm{s}}(\omega)$. Note that the orthogonality properties and normalization defined by Eqs. (3) and (4) yield $\left[\mathcal{M}_{\mathrm{LM}}^{\mathrm{s}}\right]=\left[I_{n_{\mathrm{LM}}^{\mathrm{s}}}\right]$ and $\left[\mathcal{K}_{\mathrm{LM}}^{\mathrm{s}}\right]=\left[\Lambda_{\mathrm{LM}}^{\mathrm{s}}\right]$ in which $\left[\Lambda_{\mathrm{LM}}^{\mathrm{s}}\right]_{\alpha \beta}=\lambda_{\alpha}^{\mathrm{s}} \delta_{\alpha \beta}$ is a diagonal $n_{\mathrm{LM}}^{\mathrm{s}} \times n_{\mathrm{LM}}^{\mathrm{s}}$ matrix.

\section{Multilevel basis}

In this section, a methodology is presented to construct a new basis for $₫(\omega)$, different from elastic modes. For constructing such a basis, the approach relies on a spatial filtering to decompose the set of the elastic modes into two sets of linearly independent displacement vectors that are no longer elastic modes. The two sets of displacement vectors are characterized by a 
polynomial degree. First, a set of spatial filtering functions is constructed as a set of mutually orthogonal multivariate polynomials, with respect to a given metric related to the structural mass distribution on $\Omega_{\mathrm{s}}$. Let $\left[\mathbb{M}^{\text {lump }}\right]$ be the lumped mass matrix derived from the mass matrix of the structure. The discrete mass $M_{\gamma}=\left[\mathbb{M}^{\text {lump }}\right]_{J_{\gamma} J_{\gamma}}$ is associated with the $J_{\gamma}$-th degree of freedom that corresponds to one of the three translational displacements located at the $\gamma$-th node of the finite element mesh and located at position $\mathbf{x}^{\gamma}$. Let [m lump ] be the diagonal $n_{\text {nodes }} \times n_{\text {nodes }}$ real-valued matrix defined by [m $\left.{ }^{\text {lump }}\right]_{\gamma \widetilde{\gamma}}=M_{\gamma} \delta_{\gamma \widetilde{\gamma}}$ of the lumped mass at the $n_{\text {nodes }}$ nodes of the finite-element mesh of the structure. In this work, the set of spatial filtering functions is chosen as the multivariate three-dimensional polynomials $p_{1}, p_{2}, \ldots, p_{n_{\text {mon }}}$ defined on $\Omega_{\mathrm{s}}$ such that

$$
\sum_{\gamma=1}^{n_{\text {nodes }}} M_{\gamma} p_{\ell_{1}}\left(\mathbf{x}^{\gamma}\right) p_{\ell_{2}}\left(\mathbf{x}^{\gamma}\right)=\delta_{\ell_{1} \ell_{2}} .
$$

Consequently, the shape of each spatial filtering function is weighted by the spatial mass distribution of the structure. Such set of spatial filtering function can then represent the displacements of separate components or assembled components of the structure as an automobile. The large wavelength of an elastic mode and a spatially localized elastic mode can be written as a sum of spatial filtering functions with a relatively small polynomial degree in contrast to short wavelength elastic modes, which can only be represented by sufficiently high degrees (for instance, in the presented application, the maximum degree that is considered is 40). Hence, the polynomial degree of the spatial filtering functions can be used to characterized the level of complexity for the vector space spanned by a set of elastic modes of the structure. The more the polynomial degree of the spatial filtering functions is high, the more the complexity level is important. Hereinafter, we will present the construction of low-complexity-level displacement basis and its complementary counterpart into the set of the high-complexity-level displacement basis. Gathering the two bases together yields the Multi-complexity-level displacements basis that will be called the Multilevel basis. Such an approach has also been introduced in [80, 81], but in this paper, the presentation, the developments, and the formulated interpretations are different. Note that such an approach is only used for the elastic modes of the structure and not for the acoustic cavity (the air in the cockpit of the automobile) because the acoustic cavity is homogeneous with regards to such a complexity characterization.

\subsection{Computation of the filtering-functions values at the finite-element nodes}

Let $m_{1}, m_{2}, \ldots$ be the real-valued multivariate monomials defined on $\Omega_{\mathrm{s}}$ such that, for all $\mathbf{x}=\left(x_{1}, x_{2}, x_{3}\right)$ in $\Omega_{\mathrm{s}}$,

$$
m_{k}(\mathbf{x})=\left(x_{1}\right)^{\alpha_{k}^{1}-\alpha_{k}^{2}}\left(x_{2}\right)^{\alpha_{k}^{2}-\alpha_{k}^{3}}\left(x_{3}\right)^{\alpha_{k}^{3}},
$$

in which $\boldsymbol{\alpha}_{k}=\left(\alpha_{k}^{1}, \alpha_{k}^{2}, \alpha_{k}^{3}\right)$ is the $k$-th three-dimensional multi-index such that $0<\left|\boldsymbol{\alpha}_{1}\right| \leq\left|\boldsymbol{\alpha}_{2}\right| \ldots$ with $\left|\boldsymbol{\alpha}_{k}\right|=\alpha_{k}^{1}+\alpha_{k}^{2}+\alpha_{k}^{3}$ and such that $0 \leq \alpha_{k}^{3} \leq \alpha_{k}^{2} \leq \alpha_{k}^{1} \leq d$. The number of such monomials with degree less or equal to $d$ is $n_{\text {mon }}=(d+1)(d+2)(d+3) / 6$.

Let $[p]$ be the $n_{\text {nodes }} \times n_{\text {mon }}$ matrix of the polynomial values at the nodes of the mesh such that $[p]_{\gamma \ell}=p_{\ell}\left(\mathbf{x}^{\gamma}\right)$ and let $[m]$ be the real $n_{\text {nodes }} \times n_{\text {mon }}$ matrix of the monomial values at the nodes of the mesh such that $[m]_{\gamma k}=m_{k}\left(\mathbf{x}^{\gamma}\right)$. We then have

$$
[p]=[m][s],
$$

in which $[s]$ is an upper triangular matrix that has to be constructed. Furthermore, Eq. (9) can be 
rewritten as

$$
[p]^{T}\left[\mathrm{~m}^{\text {lump }}\right][p]=\left[I_{n_{\operatorname{mon}}}\right] .
$$

The nodal values of polynomials $p_{1}, \ldots, p_{n_{\operatorname{mon}}}$ can then be calculated by $[p]=\left[\mathrm{m}^{\text {lump }}\right]^{-1 / 2}[q]$ where $[q]$ is an orthogonal matrix, which can directly be computed as the thin (economy size) QR decomposition $[q][r]$ of matrix $\left[m^{\text {lump }}\right]^{1 / 2}[m]$, which shows that $[m]=[p][r]$. Consequently, we have $[s]=[r]^{-1}$. Nevertheless, the computation of $[p]$ is not required for efficiently carrying out the filtering approach presented into the next sections, which avoids the numerical cost to compute inverse matrix $[r]^{-1}$ (i.e the computation of $[s]$ ) and also avoids to store $[r]$. Only the computation of $[q]$ is required.

\subsection{Finite-element projection matrix associated with the filtering functions}

Let $u^{1}, u^{2}$, and $u^{3}$ be the $\mathbb{R}^{n_{\text {nodes }}}$ vectors of all the degrees of freedom that correspond to the translational displacements into directions $\mathbf{e}_{1}, \mathbf{e}_{2}$, and $\mathbf{e}_{3}$ for the nodes of the finite element mesh. Let $w$ be the vector of all the degrees of freedom that are not translational displacements. Let $u$ be the vector of all the degrees of freedom that, consequently, gathers all values of $u^{1}, u^{2}, u^{3}$, and $w$. Assembling $u^{1}, u^{2}, u^{3}$, and $w$ into $u$ yields

$$
u=[R] w+\sum_{j=1}^{3}\left[T_{j}\right] \boldsymbol{u}^{j},
$$

in which, for all $j$ in $\{1,2,3\}$, the entries of the $n_{\text {dof }}^{\mathrm{s}} \times n_{\text {nodes }}$ matrix $\left[T_{j}\right]$ are 0 or 1 only and such that, for all $j$ and $j^{\prime}$ in $\{1,2,3\}$,

$$
\begin{aligned}
& {\left[T_{j}\right]^{T}\left[T_{j^{\prime}}\right]=\delta_{j j^{\prime}}\left[I_{n_{\text {nodes }}}\right],} \\
& {\left[T_{j}\right]^{T}[R]=[0] .}
\end{aligned}
$$

For all $j$ in $\{1,2,3\}$, it can then be deduced that

$$
u^{j}=\left[T_{j}\right]^{T} u .
$$

In addition, since $\left[\mathbb{M}^{\text {lump }}\right]$ is diagonal, then, for all $j$ and $j^{\prime}$ in $\{1,2,3\}$, we also have

$$
\left[T_{j}\right]^{T}\left[\mathbb{M}^{\text {lump }}\right]\left[T_{j^{\prime}}\right]=\delta_{j j^{\prime}}\left[\mathrm{m}^{\text {lump }}\right] .
$$

For $j$ in $\{1,2,3\}$, let $\mathbf{u}^{j}=[p] \mathbf{c}^{j}$ be the polynomial approximation of vector $u^{j}$ such that $\mathbf{c}^{j}$ in $\mathbb{R}^{n_{\text {mon }}}$ minimizes $\mathbf{c} \mapsto\left\|[p] \mathbf{c}-u^{j}\right\|^{2}$. The solution of this least-square minimization problem is well known and can be written, using Eqs. (10) and (11), as

$$
\mathbf{c}^{j}=[p]^{T}\left[\mathrm{~m}^{\text {lump }}\right]\left[T_{j}\right]^{T} \text { u. }
$$

Consequently, for all $j$ in $\{1,2,3\}$, the polynomial approximation $\mathbf{u}^{j}$ of vector $\mho^{j}$ is such that

$$
\mathbf{u}^{j}=[p][p]^{T}\left[m^{\text {lump }}\right]\left[T_{j}\right]^{T} u .
$$

Let us introduce the polynomial approximation $\mathbf{u}$ of the vector of the degrees of freedom $u$ constructed by replacing $u^{j}$ by its polynomial approximation $\mathbf{u}^{j}$ and by replacing the non-translational 
degrees of freedom $w$ by the null vector. We then have

$$
\mathbf{u}=[\mathbb{P}] \boldsymbol{u},
$$

in which the $n_{\mathrm{dof}}^{\mathrm{s}} \times n_{\mathrm{dof}}^{\mathrm{s}}$ matrix $[\mathbb{P}]$ is defined by

$$
[\mathbb{P}]=\sum_{j=1}^{3}\left[T_{j}\right][p][p]^{T}\left[\mathrm{~m}^{\text {lump }}\right]\left[T_{j}\right]^{T} .
$$

Consequently, $[\mathbb{P}]$ can be computed by the usual assembling algorithm of the finite element method for the element matrix $[p][p]^{T}\left[\mathrm{~m}^{\text {lump }}\right]$. In addition, the null space of $[\mathbb{P}]$ is reduced to $\{\mathbf{0}\}:[\mathbb{P}] \varangle_{0}=\mathbf{0}$ implies $\varangle_{0}=\mathbf{0}$, because for any non-zero vector $\varangle_{0}$ and for any vector $\mathbb{W}$, we have

$$
u_{0}=[R] w+\sum_{j=1}^{3}\left[T_{j}\right] u_{0}^{j} \operatorname{with}[p]^{T}\left[\mathrm{~m}^{\text {lump }}\right] u_{0}^{j}=\mathbf{0} .
$$

Consequently, matrix $[\mathbb{P}]$ is referred as the matrix of the finite-element projection associated with the spatial filtering.

\subsection{Projected mass matrix}

An unusual mass matrix is introduced and defined as the finite-element projection on the filtering functions $[\mathbb{P}]^{T}\left[\mathbb{M}^{\mathrm{s}}\right][\mathbb{P}]$ of mass matrix $\left[\mathbb{M}^{\mathrm{s}}\right]$. It will be used for setting up an unusual eigenvalue problem from which the low- and high-complex-level displacement bases will be constructed. Note that $n_{\mathrm{dof}}^{\mathrm{s}}$ can be equal to several millions and consequently, the full matrix $[\mathbb{P}]$ cannot be stored in the random access memory (RAM) or even on a hard disk. The computation of $[\mathbb{P}]^{T}\left[\mathbb{M}^{\mathrm{s}}\right][\mathbb{P}]$ is replaced by the computation of $\left[\mathbb{M}^{\text {proj }}\right]=[\mathbb{P}]^{T}\left[\mathbb{M}^{\text {lump }}\right][\mathbb{P}]$ in order to take advantage of orthogonality relations of polynomials $[p]$. Replacing $[\mathbb{P}]$ defined by Eq. [13), using Eq. (12) and then Eq. $\left[10\right.$, and introducing matrix $[b]=\left[\mathrm{m}^{\text {lump }}\right]^{1 / 2}[q]$, allow matrix $\left[\mathbb{M}^{\text {proj }}\right]$ to be written as

$$
\left[\mathbb{M}^{\text {proj }}\right]=\sum_{j=1}^{3}\left[T_{j}\right][b][b]^{T}\left[T_{j}\right]^{T} .
$$

Consequently, the projected matrix $\left[\mathbb{M}^{\text {proj }}\right]$ can be constructed by using the usual finite-element assembling algorithm applied to the $n_{\text {nodes }} \times n_{\text {nodes }}$ matrix $[b][b]^{T}$. Furthermore, Eq. (14) can be rewritten as

$$
\left[\mathbb{M}^{\text {proj }}\right]=[\mathbb{N}][\mathbb{N}]^{T},
$$

where the $n_{\text {dof }}^{\mathrm{s}} \times n_{\text {mon }}$ matrix [N] is defined by

$$
[\mathbb{N}]=\left[\left[T_{1}\right][b] \quad\left[T_{2}\right][b] \quad\left[T_{3}\right][b]\right] .
$$

\subsection{Conditions on the reduced-order basis for the filtering}

Let $N$ be a given positive integer such that $N \leq n_{\text {dof }}^{\mathrm{s}}$ and let [U] be a given $n_{\text {dof }}^{\mathrm{s}} \times N$ matrix for which the columns is a set of linearly independent finite-element displacements that are mutually orthogonal with respect to the metric defined by $\left[\mathbb{M}^{\mathrm{s}}\right]$ and $\left[\mathbb{K}^{\mathrm{s}}\right]$, such that

$$
[\mathbb{U}]^{T}\left[\mathbb{K}^{\mathrm{s}}\right][\mathbb{U}]=[\Lambda], \quad[\mathbb{U}]^{T}\left[\mathbb{M}^{\mathrm{s}}\right][\mathbb{U}]=\left[I_{N}\right],
$$


where [ $\Lambda]$ is a $N \times N$ positive-definite diagonal matrix and where $\left[I_{N}\right]$ is the $N \times N$ unit matrix. It should be noted that such orthogonality relations are also verified by matrix $\left[\Phi^{\mathrm{s}}\right]$ defined in Section 2. Nevertheless, they do not imply that $\left[\mathbb{K}^{\mathrm{s}}\right][\mathbb{U}]=\left[\mathbb{M}^{\mathrm{s}}\right][\mathbb{U}][\Lambda]$.

The spatial filtering method that is presented in this paper can be applied to any reducedorder basis represented by such a matrix $[\mathbb{U}]$ that fulfills relations in Eq. $\left[15\right.$, such as $\left[\Phi^{\mathrm{s}}\right]$ for instance. Applying the following method with $[\mathbb{U}]=\left[\Phi^{\mathrm{s}}\right]$ will be carried out in order to define the first spatial filtering into the next sections.

\subsection{Reduced-order projected mass matrix}

The reduced-order projected mass matrix $\left[\mathcal{M}^{\mathrm{proj}}\right]$ is then introduced as the $N \times N$ matrix defined by

$$
\left[\mathcal{M}^{\text {proj }}\right]=[\mathbb{U}]^{T}\left[\mathbb{M}^{\text {proj }}\right][\mathbb{U}] .
$$

Note that since $\left[\mathbb{M}^{\text {proj }}\right]$ is positive but not definite, then $\left[\mathcal{M}^{\text {proj }}\right]$ is also positive but not definite. Using Eq. 14] yields

$$
\left[\mathcal{M}^{\text {proj }}\right]=[\mathcal{N}][\mathcal{N}]^{T},
$$

in which the $N \times n_{\text {mon }}$ matrix $[\mathcal{N}]$ is written as

$$
\begin{aligned}
& {[\mathcal{N}]=[\mathbb{U}]^{T}[\mathbb{N}],} \\
& \left.=\left[\begin{array}{lll}
{\left[\mathbb{U}_{1}\right.} & ]^{T}[b] & {\left[\mathbb{U}_{2}\right.}
\end{array}\right]^{T}[b] \quad\left[\mathbb{U}_{3}\right]^{T}[b]\right],
\end{aligned}
$$

in which, for $j$ in $\{1,2,3\},\left[\mathbb{U}_{j}\right]=\left[T_{j}\right]^{T}[\mathbb{U}]$ is a $n_{\text {nodes }} \times N$ matrix. From a numerical programming aspect, matrix $\left[\mathbb{U}_{j}\right]$ is constructed by removing in $[\mathbb{U}]$ all the rows that do not correspond to the degrees of freedom of translational displacements into direction $\mathbf{e}_{j}$.

\subsection{Construction of the low-complexity-level displacement basis}

Let $\mathcal{S}$ be the vector space that is spanned by columns of [U] . Let $\mathcal{S}^{\text {low }}$ be the subspace of $\mathcal{S}$ that is defined as the vector space of all the vectors of finite element displacements $\vee$ in $\mathcal{S}$ with non-zero kinetic energy $\frac{1}{2} v^{T}\left[\mathbb{M}^{\text {proj }}\right] \vee$. Let $n^{\text {low }}$ be the dimension of $\mathcal{S}^{\text {low }}$, which is assumed to be spanned by a set of $n^{\text {low }}$ linearly independent vectors $\left\|_{1}^{\text {low }} \ldots\right\|_{n^{\text {low }}}^{\text {low }}$ of dimension $n_{\text {dof }}^{\mathrm{s}}$. Since $\mathcal{S}^{\text {low }}$ is a subspace of $\mathcal{S}$ by construction, then $\varkappa_{\alpha}^{\text {low }}$ can be written, for all $\alpha$ in $\left\{1, \ldots, n^{\text {low }}\right\}$, as

$$
u_{\alpha}^{\text {low }}=[\mathbb{U}]\left[\Phi^{\text {proj }}\right] \varphi_{\alpha}^{\text {low }},
$$

where $\varphi_{1}^{\text {low }}, \ldots, \varphi_{n^{\text {low }}}^{\text {low }}$ are linearly independent vectors of dimension $n^{\text {low }}$ and $\left[\Phi^{\text {proj }}\right]$ is a $N \times n^{\text {low }}$ rectangular matrix such that $\left[\Phi^{\text {proj }}\right]^{T}$ corresponds to the orthogonal projection operator from $\mathcal{S}$ into $\mathcal{S}^{\text {low }}$ for a given metric that is defined hereinafter. This projection corresponds to a so-called Rayleigh-Ritz approximation.

The objective of this section is to construct matrix $\left[\Phi^{\text {proj }}\right]$ and the set of linearly independent vectors $\varkappa_{1}^{\text {low }} \ldots \otimes_{n^{\text {low }}}^{\text {low }}$.

Let us first consider the following generalized eigenvalue problem: find $\varphi_{\alpha}^{\text {proj }}$ in $\mathbb{R}^{N}$ and $\lambda_{\alpha}^{\text {proj }}>0$ such that

$$
[\Lambda] \varphi_{\alpha}^{\text {proj }}=\lambda_{\alpha}^{\text {proj }}\left[\mathcal{M}^{\text {proj }}\right] \varphi_{\alpha}^{\text {proj }} .
$$

This first eigenvalue problem involves a Rayleigh-Ritz projection of the original stiffness matrix $\left[\mathbb{K}^{s}\right]$ and the projected stiffness matrix $\left[\mathbb{M}^{\text {proj }}\right]$. It allows for defining the set of the $n^{\text {low }}$ linearly 
independent finite element vectors $\left[\mathbb{U}^{\text {proj }}\right]=\left[\mathbb{u}_{1}^{\text {proj }} \ldots \mathbb{u}_{n^{\text {low }}}^{\text {proj }}\right]$ such that, for all $\alpha$ in $\left\{1, \ldots, n^{\text {low }}\right\}$, $u_{\alpha}^{\text {proj }}$ is written as

$$
u_{\alpha}^{\mathrm{proj}}=[\mathbb{U}] \varphi_{\alpha}^{\mathrm{proj}} .
$$

Since matrix $\left[\mathcal{M}^{\text {proj }}\right]$ is positive semi-definite, then there are infinite eigenvalues. The eigenvalues are sorted in ascending order $0<\lambda_{1}^{\text {proj }} \leq \ldots \leq \lambda_{n^{\text {ow }}}^{\text {proj }}<\ldots \leq \lambda_{n}^{\text {proj }}$ in which rank $n^{\text {low }}$ is such that $\lambda_{\alpha}^{\text {proj }}$ is not finite for any $\alpha>n^{\text {low }}$. Note that computing matrix $\left[\mathcal{M}^{\text {proj }}\right]$ is not required. By using Eq. [16, the thin SVD of matrix $[\Lambda]^{-1 / 2}[\mathcal{N}]$ is written as $[\Lambda]^{-1 / 2}[\mathcal{N}]=\left[U^{\text {proj }}\right]\left[\Sigma^{\text {proj }}\right]\left[V^{\text {proj }}\right]^{T}$ in which $\left[U^{\text {proj }}\right]$ is a rectangular $N \times n^{\text {low }}$ matrix with $\left[U^{\text {proj }}\right]^{T}\left[U^{\text {proj }}\right]=\left[I_{n^{\text {low }}}\right]$, where $\left[V^{\text {proj }}\right]$ is a rectangular $n_{\text {mon }} \times n^{\text {low }}$ matrix with $\left[V^{\text {proj }}\right]^{T}\left[V^{\text {proj }}\right]=\left[I_{n^{\text {low }}}\right]\left(\left[V^{\text {proj }}\right]\right.$ does not need to be computed), and where $\left[\Sigma^{\text {proj}}\right]$ is a diagonal positive-definite $n^{\text {low }} \times n^{\text {low }}$ matrix.

For all $\alpha=1, \ldots, n^{\text {low }}$, we have $\lambda_{\alpha}^{\text {proj }}=\left(\left[\Sigma^{\text {proj }}\right]_{\alpha \alpha}\right)^{-2}$ and $\left[\Phi^{\text {proj }}\right]=[\Lambda]^{-1 / 2}\left[U^{\text {proj }}\right]\left[\Sigma^{\text {proj }}\right]$ where $\left[\Phi^{\text {proj }}\right]$ is the $N \times n^{\text {low }}$ modal matrix $\left[\Phi^{\text {proj }}\right]=\left[\begin{array}{lll}\varphi_{1}^{\text {proj }} & \ldots \varphi_{n^{\text {low }}}^{\text {proj }}\end{array}\right]$ Furthermore, we also have the two orthogonality and normalization relations,

$$
\begin{aligned}
& {\left[\Phi^{\mathrm{proj}}\right]^{T}[\Lambda]\left[\Phi^{\mathrm{proj}}\right]=\left[\Sigma^{\mathrm{proj}}\right],} \\
& {\left[\Phi^{\mathrm{proj}}\right]^{T}\left[\mathcal{M}^{\mathrm{proj}}\right]\left[\Phi^{\mathrm{proj}}\right]=\left[I_{n^{\mathrm{low}}}\right] .}
\end{aligned}
$$

A second eigenvalue problem is then solved for eventually constructing the low-complexity-level displacement basis. It consists in finding $\varphi_{\alpha}^{\text {low }}$ in $\mathbb{R}^{n^{\text {low }}}$ and $\lambda_{\alpha}^{\text {low }}>0$ such that

$$
\left[\Sigma^{\mathrm{proj}}\right] \varphi_{\alpha}^{\text {low }}=\lambda_{\alpha}^{\text {low }}\left[\Phi^{\mathrm{proj}}\right]^{T}\left[\Phi^{\mathrm{proj}}\right] \varphi_{\alpha}^{\text {low }} .
$$

This eigenvalue problem involves again a Rayleigh-Ritz projection of the original stiffness and mass matrices $\left[\mathbb{K}^{s}\right]$ and $\left[\mathbb{M}^{s}\right]$. It allows for defining the low-level-complexity displacement ba-

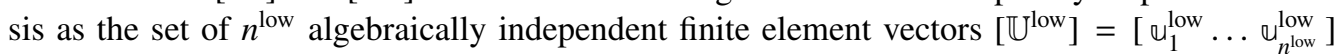
defined, for all $\alpha$ in $\left\{1, \ldots, n^{\text {low }}\right\}$, by

$$
\mathbb{u}_{\alpha}^{\text {low }}=[\mathbb{U}]\left[\Phi^{\text {proj }}\right] \varphi_{\alpha}^{\text {low }} .
$$

The $n^{\text {low }}$ eigenvalues $0<\lambda_{1}^{\text {low }} \leq \ldots \leq \ldots \leq \lambda_{n^{\text {low }}}^{\text {low }}$ of the generalized eigenvalue problem defined in Eq. (17) are finite and positive and the associated eigenvectors $\varphi_{1}^{\text {low }}, \ldots, \varphi_{n^{\text {low }}}^{\text {low }}$ are assumed to be normalized with respect to matrix $\left[\Phi^{\text {proj }}\right]^{T}\left[\Phi^{\text {proj }}\right]$.

Once again, computing matrix $\left[\Phi^{\text {proj }}\right]^{T}\left[\Phi^{\text {proj }}\right]$ is not required when $n^{\text {low }}$ is large. Using the SVD allows us to write $\left[\Sigma^{\text {proj }}\right]^{-1 / 2}\left[\Phi^{\text {proj }}\right]^{T}=\left[U^{\text {low }}\right]\left[\Sigma^{\text {low }}\right]\left[V^{\text {low }}\right]^{T}$ in which $\left[U^{\text {low }}\right]$ and $\left[V^{\text {low }}\right]$ are two $n^{\text {low }} \times n^{\text {low }}$ unitary matrices and [ $\left.\Sigma^{\text {low }}\right]$ is a diagonal positive-definite $n^{\text {low }} \times n^{\text {low }}$ matrix. We then have $\lambda_{\alpha}^{\text {low }}=\left(\left[\Sigma^{\text {low }}\right]_{\alpha \alpha}\right)^{-2}$ for $\alpha$ in $\left\{1, \ldots, n^{\text {low }}\right\}$ and $\left[\Phi^{\text {low }}\right]=\left[\Sigma^{\text {proj }}\right]^{-1 / 2}\left[U^{\text {low }}\right]\left[\Sigma^{\text {low }}\right]$ where $\left[\Phi^{\text {low }}\right]$ is the $n^{\text {low }} \times n^{\text {low }}$ modal matrix $\left[\Phi^{\text {low }}\right]=\left[\varphi_{1}^{\text {low }} \ldots \varphi_{n^{\text {low }}}^{\text {low }}\right]$. Furthermore, we also have the two orthogonality and normalization relations

$$
\begin{array}{r}
{\left[\Phi^{\mathrm{low}}\right]^{T}\left[\Phi^{\mathrm{proj}}\right]^{T}[\Lambda]\left[\Phi^{\mathrm{proj}}\right]\left[\Phi^{\mathrm{low}}\right]=\left[\Lambda^{\mathrm{low}}\right],} \\
{\left[\Phi^{\mathrm{low}}\right]^{T}\left[\Phi^{\mathrm{proj}}\right]^{T}\left[\Phi^{\mathrm{proj}}\right]\left[\Phi^{\mathrm{low}}\right]=\left[I_{n^{\mathrm{low}}}\right],}
\end{array}
$$

where $\left[\Lambda^{\text {low }}\right]=\left[\Sigma^{\text {low }}\right]^{-2}$ is a diagonal matrix. Note that such low-level-complexity displacement basis spans the same vector space $\mathcal{S}^{\text {low }}$ as the set of the $n^{\text {low }}$ linearly independent vectors 
$\left\{\mathrm{u}_{1}^{\text {proj }}, \ldots, \mathrm{u}_{n^{\mathrm{low}}}^{\text {proj }}\right\}$. It should also be noted that

$$
\begin{aligned}
& {\left[\mathbb{U}^{\text {low }}\right]^{T}\left[\mathbb{K}^{\mathrm{s}}\right]\left[\mathbb{U}^{\text {low }}\right]=\left[\Lambda^{\text {low }}\right],} \\
& {\left[\mathbb{U}^{\text {low }}\right]^{T}\left[\mathbb{M}^{\mathrm{s}}\right]\left[\mathbb{U}^{\text {low }}\right]=\left[I_{n^{\text {low }}}\right],}
\end{aligned}
$$

while there is only one orthogonality relation that can be built for $\left[\mathbb{U}^{\text {proj }}\right]$,

$$
\left[\mathbb{U}^{\text {proj }}\right]^{T}\left[\mathbb{K}^{\mathrm{s}}\right]\left[\mathbb{U}^{\text {proj }}\right]=\left[\Lambda^{\text {proj }}\right] .
$$

Consequently, [ $\mathbb{U}^{\text {low }}$ ] fulfills orthogonality relations in Eq. 20 and then the spatial filtering

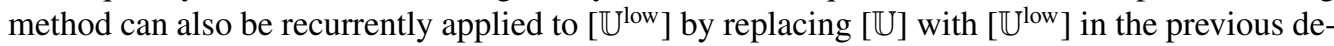
velopments, which is not possible for [ $\left.\mathbb{U}^{\mathrm{proj}}\right]$. Such a recurrence will be used for introducing the second spatial filtering. Despite relations in Eq. (20) seem to be very similar to the relations in Eq. (3), it should be noted that, in general, $\lambda_{\alpha}^{\text {low }} \neq \lambda_{\alpha}^{\mathrm{s}}$ and $\mu_{\alpha}^{\text {low }} \neq \varphi_{\alpha}^{\mathrm{s}}$ for all $\alpha$ in $\left\{1, \ldots, n^{\text {low }}\right\}$. Consequently, in general, the low-complexity-level displacement basis [ $\mathbb{U}^{\text {proj }}$ ] does not correspond to elastic modes. Furthermore, it will be more convenient into the next sections to introduce matrix $\left[Q^{\text {low }}\right]=\left[\Phi^{\text {proj }}\right]\left[\Phi^{\text {low }}\right]$. By using Eq. (19), we then have

$$
\begin{aligned}
& {\left[Q^{\text {low }}\right]^{T}\left[Q^{\text {low }}\right]=\left[I_{n^{\text {low }}}\right],} \\
& {\left[\mathbb{U}^{\text {low }}\right]=[\mathbb{U}]\left[Q^{\text {low }}\right] .}
\end{aligned}
$$

\subsection{Construction of the high-complexity-level displacement basis}

Since $n^{\text {low }}$ is usually very small with respect to $N$, then vector space $\mathcal{S}^{\text {low }}$ might not allow an accurate representation of any finite element solution $u(\omega)$ that is represented by the basis made up of the columns of [U]]. It is the reason why a second displacement basis is introduced and that is such that it spans the vector space $\mathcal{S}^{\text {high }}$ defined as the complement vector space of $\mathcal{S}^{\text {low }}$ into $\mathcal{S}$ with respect to the metric associated with mass matrix $\left[\mathbb{M}^{\mathrm{s}}\right]$. Let $n^{\text {high }}=N-n^{\text {low }}$ and let $\left[\mathbb{U}^{\text {high }}\right]=\left[\mathbb{U}_{1}^{\text {high }} \ldots \mathbb{U}_{n^{\text {high }}}^{\text {high }}\right]$ be the $n_{\text {dof }}^{\text {s }} \times n^{\text {high }}$ matrix whose columns form a set of mutually linearly independent vectors $\mathbb{u}_{1}^{\text {high }}, \ldots, \mathbb{U}_{n^{\text {high }}}^{\text {high }}$ that span $\mathcal{S}^{\text {high }}$. Hereinafter, $\left[\mathbb{U}^{\text {high }}\right]$ will be referred as the high-complexity-level displacement basis. As $\mathcal{S}^{\text {high }}$ is a subspace of $\mathcal{S}^{\text {s }}$ (by construction), then for all $\alpha$ in $\left\{1, \ldots, n^{\text {high }}\right\}, \varkappa_{\alpha}^{\text {high }}$ can be written as

$$
\mathbb{U}_{\alpha}^{\text {high }}=[\mathbb{U}]\left[\Phi^{\mathrm{comp}}\right] \varphi_{\alpha}^{\mathrm{high}},
$$

in which $\varphi_{1}^{\text {high }}, \ldots, \varphi_{n^{\text {high }}}^{\text {high }}$ are linearly independent vectors belonging to $\mathbb{R}^{n^{\text {high }}}$ and where [ $\left.\Phi^{\text {comp }}\right]$ is a $N \times n^{\text {high }}$ rectangular matrix such that

$$
\left[\Phi^{\mathrm{comp}}\right]^{T}\left[\Phi^{\mathrm{comp}}\right]=\left[I_{n^{\text {high }}}\right],
$$

whose construction is defined hereinafter. The orthogonality property is introduced for guarantying the recursive constructions of the low- and high -complexity level displacement bases at different levels (see Section 4).

Since by construction, $\mathbb{u}_{\alpha}^{\text {nigh }}$ is normal (for the metric defined by matrix $\left[\mathbb{M}^{\mathrm{s}}\right]$ ) to subspace $\mathcal{S}^{\text {proj }}$, for all $\alpha$ in $\left\{1, \ldots, n^{\text {high }}\right\}$, we have

$$
\left[\mathbb{U}^{\text {proj }}\right]^{T}\left[\mathbb{M}^{\mathrm{s}}\right] \mathrm{u}_{\alpha}^{\text {high }}=\mathbf{0} .
$$


Since $[\mathbb{U}]^{T}\left[\mathbb{M}^{\mathrm{s}}\right][\mathbb{U}]=\left[I_{N}\right]$ then Eq. 24 is rewritten as

$$
\left[\Phi^{\mathrm{proj}}\right]^{T}\left[\Phi^{\mathrm{comp}}\right] \varphi_{\alpha}^{\text {high }}=\mathbf{0} .
$$

Since Eq. 25] is verified for linearly independent vectors $\varphi_{1}^{\text {high }}, \ldots, \varphi_{n^{\text {high }}}^{\text {high }} \mathbb{R}^{n^{\text {high }}}$, we have

$$
\left[\Phi^{\mathrm{proj}}\right]^{T}\left[\Phi^{\mathrm{comp}}\right]=\left[0_{n^{\mathrm{low}} \times n^{\mathrm{high}}}\right] .
$$

Taking into account Eqs. (23) and (26), the matrix [ $\left.\Phi^{\text {comp }}\right]$ can be defined as the real matrix whose columns are the right-singular vectors associated with the $n^{\text {high }}$ zero singular values of the SVD of matrix $\left[\Phi^{\text {proj }}\right]^{T}$. The linearly independent vectors $\varphi_{1}^{\text {high }}, \ldots, \varphi_{n^{\text {high }}}^{\text {high }}$ in $\mathbb{R}^{n^{\text {high }}}$ can be defined as the solutions of the following generalized eigenvalue problem,

$$
\left[\Phi^{\mathrm{comp}}\right]^{T}[\Lambda]\left[\Phi^{\mathrm{comp}}\right] \varphi_{\alpha}^{\mathrm{high}}=\lambda_{\alpha}^{\mathrm{high}} \varphi_{\alpha}^{\text {high }} .
$$

Consequently, we have

$$
\begin{aligned}
& {\left[\mathbb{U}^{\text {high }}\right]^{T}\left[\mathbb{K}^{\mathrm{s}}\right]\left[\mathbb{U}^{\text {high }}\right]=\left[\Lambda^{\text {high }}\right],} \\
& {\left[\mathbb{U}^{\text {high }}\right]^{T}\left[\mathbb{M}^{\mathrm{s}}\right]\left[\mathbb{U}^{\text {high }}\right]=\left[I_{n^{\text {high }}}\right],}
\end{aligned}
$$

in which $\left[\Lambda^{\text {high }}\right]$ is the positive-definite diagonal matrix of the eigenvalues $\lambda_{1}^{\text {high }}, \ldots, \lambda_{n^{\text {high }}}^{\text {high }}$ and where $\left[\Phi^{\text {high }}\right]=\left[\varphi_{1}^{\text {high }} \ldots \varphi_{n^{\text {high }}}^{\text {high }}\right]$ is such that

$$
\left[\Phi^{\text {high }}\right]^{T}\left[\Phi^{\text {high }}\right]=\left[I_{N}\right] .
$$

Furthermore, it will be more convenient into the next sections to introduce matrix $\left[Q^{\text {high }}\right]=$ $\left[\Phi^{\text {comp }}\right]\left[\Phi^{\text {high }}\right]$. We then have

$$
\left[\mathbb{U}^{\text {high }}\right]=[\mathbb{U}]\left[Q^{\text {high }}\right] .
$$

Using Eqs. (23), 26), and (28) yields

$$
\begin{aligned}
& {\left[Q^{\text {high }}\right]^{T}\left[Q^{\text {high }}\right]=\left[I_{n^{\text {high }}}\right],} \\
& {\left[Q^{\text {low }}\right]^{T}\left[Q^{\text {high }}\right]=\left[0_{n^{\text {low }} \times n^{\text {high }}}\right] .}
\end{aligned}
$$

Similarly to the explanations given in Section 3.6, since [ $\mathbb{U}^{\text {high }}$ ] fulfills Eq. (27), the spatial filtering method can be recurrently also applied to [ $\left.\mathbb{U}^{\text {high }}\right]$ by replacing [U] with $\left[\mathbb{U}^{\text {high }}\right]$ in the previous developments. Such a recurrence will be used in Section 4 for introducing the second spatial filtering.

\section{Multilevel reduced-order computational model}

In this section, the previously presented methodology is used to construct a multilevel reducedorder computational model by carrying out recursively two successive spatial filterings to the elastic modes of the structure, which belong to the LF and MF bands. It is assumed that the upper- and lower-frequency bounds $\omega_{\mathrm{L}}, \omega_{\mathrm{M}}$, and $\omega_{\mathrm{H}}$ of the LF, MF, and HF bands have already been defined and are known. The strategy and the principle for the recursive two successive spatial filterings are shown in Fig. 1 . 


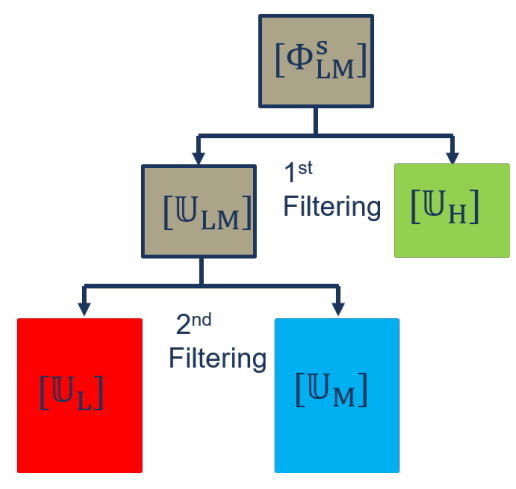

Figure 1: Two spatial filterings to construct the multilevel displacement basis

\subsection{Construction of the multilevel displacement basis for the LF and MF bands}

A first spatial filtering is carried out for $[\mathbb{U}]=\left[\Phi_{\mathrm{LM}}^{\mathrm{s}}\right]$ with a polynomial degree $d$ (see Section 3.1) equal to $d_{\mathrm{M}}$ that characterizes the complexity level of the elastic modes in the MF band in order to filter out any displacements contribution with complexity level higher than those in the MF band. This first spatial filtering yields the two displacement bases denoted by $\left[\mathbb{U}_{L M}\right]$ and $\left[\mathbb{U}_{\mathrm{H}}\right]$ that correspond to $\left[\mathbb{U}^{\text {low }}\right]$ and $\left[\mathbb{U}^{\text {high }}\right]$ introduced in Sections 3.6 and 3.6 It should be noted that the subscript $\mathrm{H}$ is used to remind that those displacements vectors have the highest complexity level, but does not refer to the HF band. The second spatial filtering is carried by replacing [U] introduced in Section 3.4 by $\left[\mathbb{U}_{\mathrm{LM}}\right]$ and with a polynomial degree $d$ equal to $d_{\mathrm{L}}$ that characterizes the complexity level of the elastic eigenvectors in the LF band. This second spatial filtering yields two displacement bases denoted by $\left[\mathbb{U}_{\mathrm{L}}\right]$ and $\left[\mathbb{U}_{\mathrm{M}}\right]$ that correspond to $\left[\mathbb{U}^{\text {low }}\right]$ and $\left[\mathbb{U}^{\text {high }}\right]$ introduced in Sections 3.6 and 3.6 Hereinafter, the complexity level of the set of elastic modes in a frequency band (LF, MF or HF) will be shortened in complexity level of the frequency band (LF, MF or HF).

\subsection{Complexity level of $L F, M F$, and $H F$ bands}

In order to determine the value $d_{\mathrm{M}}$ of $d$ for the first spatial filtering, it is proposed to study the graph of $d \mapsto \operatorname{conv}_{\mathrm{M}}(d)$ where, for all $d$ that is positive, $\operatorname{conv}_{\mathrm{M}}(d)$ is equal to the number $n^{\text {low }}$ of non-zero eigenvalues introduced in Section 3 for $[\mathbb{U}]=\left[\Phi_{\mathrm{M}}^{\mathrm{s}}\right]$. Then, the value $d_{\mathrm{M}}$ is fixed as the smallest value of $d$ such that $\operatorname{conv}_{\mathrm{M}}(d)>n_{\mathrm{H}}\left(1-\varepsilon_{\mathrm{M}}\right)$ in which $\varepsilon_{\mathrm{M}}$ is a tolerance threshold close to zero. Such a method to determine the value $d_{\mathrm{M}}$ of $d$ allows the characterization by polynomial parameter $d$ of the complexity level of the MF band. Then, using the spatial filtering with $d=d_{\mathrm{M}}$ will allow for separating every displacement contributions that have a complexity level higher than the complexity level of the MF band. To determine the value $d_{\mathrm{L}}$ of $d$ for the second spatial filtering, which allows the characterization by polynomial parameter $d$ of the complexity level of the LF domain, we use a similar method to the one proposed for determining $d_{\mathrm{M}}$. We study the graph of $d \mapsto \operatorname{conv}_{\mathrm{L}}(d)$ and the number $n^{\text {low }}$ of non-zero eigenvalues for $[\mathbb{U}]=\left[\Phi_{\mathrm{L}}^{\mathrm{s}}\right]$. Then, the value $d_{\mathrm{L}}$ is fixed as the smallest value of $d$ such that $\operatorname{conv}_{\mathrm{L}}(d)>n_{\mathrm{L}}\left(1-\varepsilon_{\mathrm{L}}\right)$ in which $\varepsilon_{\mathrm{L}}$ is a tolerance threshold close to zero. The spatial filtering with $d=d_{\mathrm{L}}$ will allow for separating the displacement contributions that have a complexity level higher than the complexity level of the LF domain. 
Furthermore, due to the limitation of the random access memory (RAM), there is a maximal value $d_{\max }$ for the polynomial parameter $d$ that can be used in practice to compute matrix [q] when performing the QR decomposition of matrix $\left[\mathrm{m}^{\text {lump }}\right]^{1 / 2}[\mathrm{~m}]$. At the time this work has been carried out, we found out that $d_{\max }=40$ on a 1500 Gigabytes RAM computer.

Such a relatively high value for $d_{\max }$ makes it possible to estimate the complexity level of the HF domain by calculating a value $d_{\mathrm{H}}$ of $\mathrm{d}$. It will be carried out by studying the graph of the function $d \mapsto \operatorname{conv}_{\mathrm{H}}(d)$ where, for all $d>0, \operatorname{conv}_{\mathrm{H}}(d)$ is equal to the number $n^{\text {low }}$ of non-zero eigenvalues introduced in Section 3 with $[\mathbb{U}]=\left[\Phi_{\mathrm{H}}^{\mathrm{s}}\right]$. Then, the value $d_{\mathrm{H}}$ is fixed as the smallest value of $d$ such that $\operatorname{conv}_{\mathrm{H}}(d)>n_{\mathrm{H}}\left(1-\varepsilon_{\mathrm{H}}\right)$ in which $\varepsilon_{\mathrm{H}}$ is a tolerance threshold much less than 1 (close to zero). Such a method to determine the value $d_{\mathrm{H}}$ of $d$ allows the characterization by polynomial parameter $d$ of the complexity level of the HF domain but it will not be used for constructing the multilevel reduced-order computational model.

\subsection{First spatial filtering}

For a given value $d_{\mathrm{M}}$ of $d$, the spatial filtering method presented in Section 3 is performed again with $[\mathbb{U}]=\left[\Phi_{\mathrm{LM}}\right]$ in order to construct two matrices $\left[\mathbb{U}^{\text {low }}\right]$ and $\left[\mathbb{U}^{\text {high }}\right]$ that are renamed as $\left[\mathbb{U}_{\mathrm{LM}}\right]$ and $\left[\mathbb{U}_{\mathrm{H}}\right]$ to avoid any confusion with the previous spatial filtering. Let $n_{\mathbb{U}_{\mathrm{LM}}}$ and $n_{\mathbb{U}_{\mathrm{H}}}$ be the number of columns of matrices $\left[\mathbb{U}_{\mathrm{LM}}\right]$ and $\left[\mathbb{U}_{\mathrm{H}}\right]$. From Eq. 22 , matrix $\left[\mathbb{U}_{\mathrm{LM}}\right]$ can be rewritten as

$$
\left[\mathbb{U}_{\mathrm{LM}}\right]=\left[\Phi_{\mathrm{LM}}^{\mathrm{s}}\right]\left[Q_{\mathrm{LM}}\right],
$$

where the matrix $\left[Q_{\mathrm{LM}}\right]$ is matrix $\left[Q^{\text {low }}\right]$ in Eq. $\left[22\right.$ with $[\mathbb{U}]=\left[\Phi_{\mathrm{LM}}^{\mathrm{s}}\right]$ and $d=d_{\mathrm{M}}$. Consequently, Eq. [21] yields

$$
\left[Q_{\mathrm{LM}}\right]^{T}\left[Q_{\mathrm{LM}}\right]=\left[I_{n_{\mathbb{L M}_{\mathrm{LM}}}}\right] .
$$

Using Eq. 29], the matrix $\left[\mathbb{U}_{H}\right]$ can be rewritten as

$$
\left[\mathbb{U}_{\mathrm{H}}\right]=\left[\Phi_{\mathrm{LM}}^{\mathrm{s}}\right]\left[Q_{\mathrm{H}}\right],
$$

in which the matrix $\left[Q_{\mathrm{H}}\right]$ is matrix $\left[Q^{\text {high }}\right]$ in Eq. (29) with [U] $=\left[\Phi_{\mathrm{LM}}^{\mathrm{s}}\right]$ and $d=d_{\mathrm{L}}$. Consequently, Eq. (30) yields

$$
\left[Q_{\mathrm{H}}\right]^{T}\left[Q_{\mathrm{H}}\right]=\left[I_{n_{\mathbb{H}_{\mathrm{H}}}}\right] .
$$

From Eq. 31), it can be deduced that

$$
\left[Q_{\mathrm{LM}}\right]^{T}\left[Q_{\mathrm{H}}\right]=\left[0_{n_{\mathbb{L M}} \times n_{\mathbb{H}_{\mathrm{H}}}}\right] .
$$

Each column of matrix $\left[\mathbb{U}_{H}\right]$ is a displacement vector with a complexity level higher than the complexity level of the MF domain. In the case of simple structures, matrix $\left[\mathbb{U}_{H}\right]$ is empty. Nevertheless, in the case of complex structures such as automobiles, matrix $\left[\mathbb{U}_{\mathrm{H}}\right]$ is never empty because local displacements are observed even in the LF band.

\subsection{Second spatial filtering}

For a given value $d_{\mathrm{L}}$ of $d$, the spatial filtering method presented in Section 3 is carried again with $[\mathbb{U}]=\left[\mathbb{U}_{\mathrm{LM}}\right]$ to construct two matrices $\left[\mathbb{U}^{\text {low }}\right]$ and $\left[\mathbb{U}^{\text {high }}\right]$ that are renamed as $\left[\mathbb{U}_{\mathrm{L}}\right]$ and $\left[\mathbb{U}_{M}\right]$ to avoid any confusion with the previous spatial filtering. Let $n_{\mathbb{U}_{L}}$ and $n_{\mathbb{U}_{M}}$ be the number of columns of matrices $\left[\mathbb{U}_{L}\right]$ and $\left[\mathbb{U}_{M}\right]$. From Eq. [22), matrix $\left[\mathbb{U}_{L}\right]$ can be rewritten as

$$
\left.\left[\mathbb{U}_{\mathrm{L}}\right]=\underset{\mathrm{LM}}{[15}\right]\left[Q_{\mathrm{LM}}\right]\left[Q_{\mathrm{L}}\right] \text {, }
$$


in which the matrix $\left[Q_{\mathrm{L}}\right]$ is matrix $\left[Q^{\text {low }}\right]$ in Eq. $\left[22\right.$ for $[\mathbb{U}]=\left[\mathbb{U}_{\mathrm{LM}}\right]$ and $d=d_{\mathrm{L}}$. Consequently, Eq. [30] yields

$$
\left[Q_{\mathrm{L}}\right]^{T}\left[Q_{\mathrm{L}}\right]=\left[I_{n_{\mathrm{L}}}\right] .
$$

Each column of matrix $\left[\mathbb{U}_{\mathrm{L}}\right]$ is a displacement vector with a complexity level of the same order than the complexity level of the LF domain. In the case for which $d_{\mathrm{L}}$ is less than $d_{\mathrm{M}}$ (that is usually the case), the subscript $L$ reminds that the vectors in the columns of $\left[\mathbb{U}_{\mathrm{L}}\right]$ gather the vectors with the lowest complexity level and does not refer to the LF band. From Eq. 29], matrix $\left[\mathbb{U}_{\mathrm{M}}\right]$ can be rewritten as

$$
\left[\mathbb{U}_{\mathrm{M}}\right]=\left[\Phi_{\mathrm{LM}}^{\mathrm{s}}\right]\left[Q_{\mathrm{LM}}\right]\left[Q_{\mathrm{M}}\right],
$$

where the matrix $\left[Q_{\mathrm{M}}\right]$ is matrix $\left[Q^{\text {high }}\right]$ in Eq. 29$]$ with $[\mathbb{U}]=\left[\mathbb{U}_{\mathrm{LM}}\right]$ and $d=d_{\mathrm{L}}$. Consequently, Eq. (30) yields

$$
\left[Q_{\mathrm{M}}\right]^{T}\left[Q_{\mathrm{M}}\right]=\left[I_{n_{\mathbb{M}_{\mathrm{M}}}}\right] .
$$

Each column of matrix $\left[\mathbb{U}_{M}\right]$ is a displacement vector with a complexity level of higher order than the complexity level of the LF band but less than the complexity level of the columns of $\left[\mathbb{U}_{H}\right]$. The subscript $M$ reminds that the vectors in the columns of $\left[\mathbb{U}_{M}\right]$ gather the vectors with a medium complexity level and does not refer to the MF domain.

\subsection{Construction of the multilevel reduced-order model in the LF and MF bands}

An approximation of displacement vector $u(\omega)$ is then written with respect to its expansion on the bases represented by matrices $\left[\mathbb{U}_{\mathrm{L}}\right],\left[\mathbb{U}_{\mathrm{M}}\right]$, and $\left[\mathbb{U}_{\mathrm{H}}\right]$,

$$
u(\omega)=\left[\mathbb{U}_{\mathrm{L}}\right] \mathbf{q}_{\mathrm{L}}^{\mathrm{s}}(\omega)+\left[\mathbb{U}_{\mathrm{M}}\right] \mathbf{q}_{\mathrm{M}}^{\mathrm{s}}(\omega)+\left[\mathbb{U}_{\mathrm{H}}\right] \mathbf{q}_{\mathrm{H}}^{\mathrm{s}}(\omega),
$$

which can be rewritten as

$$
\begin{aligned}
& u(\omega)=\left[\mathbb{U}_{\mathrm{LMH}}\right] \mathbf{q}_{\mathrm{LMH}}^{\mathrm{s}}(\omega), \\
& {\left[\mathbb{U}_{\mathrm{LMH}}\right]=\left[\left[\mathbb{U}_{\mathrm{L}}\right]\left[\mathbb{U}_{\mathrm{M}}\right]\left[\mathbb{U}_{\mathrm{H}}\right]\right], \mathbf{q}_{\mathrm{LMH}}^{\mathrm{s}}=\left[\begin{array}{r}
\mathbf{q}_{\mathrm{L}}^{\mathrm{s}}(\omega) \\
\mathbf{q}_{\mathrm{M}}^{\mathrm{s}}(\omega) \\
\mathbf{q}_{\mathrm{H}}^{\mathrm{s}}(\omega)
\end{array}\right] .}
\end{aligned}
$$

Using Eqs. [32), 36], and (38), yields

$$
\begin{aligned}
& {\left[\mathbb{U}_{\mathrm{LMH}}\right]=\left[\Phi_{\mathrm{LM}}^{\mathrm{s}}\right]\left[\mathbb{Q}_{\mathrm{LMH}}\right],} \\
& {\left[\mathbb{Q}_{\mathrm{LMH}}\right]=\left[\begin{array}{lll}
{\left[Q_{\mathrm{LM}}\right]\left[Q_{\mathrm{L}}\right]} & {\left[Q_{\mathrm{LM}}\right]\left[Q_{\mathrm{M}}\right]} & {\left[Q_{\mathrm{H}}\right]}
\end{array}\right],}
\end{aligned}
$$

and using Eqs. (33), 34, , 35), 37), and (39) yields

$$
\left[\mathbb{Q}_{\mathrm{LMH}}\right]^{T}\left[\mathbb{Q}_{\mathrm{LMH}}\right]=\left[I_{n_{\mathrm{LM}}}\right] .
$$


We then obtain the following multilevel reduced-order model,

$$
\begin{aligned}
\left(-\omega^{2}\left[M_{\mathrm{LMH}}^{\mathrm{s}}\right]\right. & \left.+i \omega\left[D_{\mathrm{LMH}}^{\mathrm{s}}\right]+\left[K_{\mathrm{LMH}}^{\mathrm{s}}\right]\right) \mathbf{q}_{\mathrm{LMH}}^{\mathrm{s}}(\omega) \\
& +\left[C_{\mathrm{LMH}}\right] \mathbf{q}^{\mathrm{f}}(\omega)=\mathbb{V}_{\mathrm{LMH}}^{\mathrm{s}}(\omega), \\
\left(-\omega^{2}\left[M^{\mathrm{f}}\right]\right. & \left.+i \omega\left[D^{\mathrm{f}}\right]+\left[K^{\mathrm{f}}\right]\right) \mathbf{q}^{\mathrm{f}}(\omega) \\
& +\omega^{2}\left[C_{\mathrm{LMH}}\right]^{T} \mathbf{q}_{\mathrm{LMH}}^{\mathrm{s}}(\omega)=\mathbf{0}
\end{aligned}
$$

in which

$$
\begin{aligned}
& {\left[M_{\mathrm{LMH}}^{\mathrm{s}}\right]=\left[\mathbb{Q}_{\mathrm{LMH}}\right]^{T}\left[\mathcal{M}_{\mathrm{LM}}^{\mathrm{s}}\right]\left[\mathbb{Q}_{\mathrm{LMH}}\right],} \\
& {\left[D_{\mathrm{LMH}}^{\mathrm{s}}\right]=\left[\mathbb{Q}_{\mathrm{LMH}}\right]^{T}\left[\mathcal{D}_{\mathrm{LM}}^{\mathrm{s}}\right]\left[\mathbb{Q}_{\mathrm{LMH}}\right],} \\
& {\left[K_{\mathrm{LMH}}^{\mathrm{s}}\right]=\left[\mathbb{Q}_{\mathrm{LMH}}\right]^{T}\left[\mathcal{K}_{\mathrm{LM}}^{\mathrm{s}}\right]\left[\mathbb{Q}_{\mathrm{LMH}}\right],} \\
& {\left[C_{\mathrm{LMH}}\right]=\left[\mathbb{Q}_{\mathrm{LMH}}\right]^{T}\left[\mathcal{C}_{\mathrm{LM}}\right],} \\
& \mathbb{G}_{\mathrm{LMH}}^{\mathrm{s}}(\omega)=\left[\mathbb{Q}_{\mathrm{LMH}}\right]^{T} \mathbf{f}_{\mathrm{LM}}^{\mathrm{s}}(\omega) .
\end{aligned}
$$

It should be noted that the orthogonality properties defined by Eq. (3) shows that $\left[M_{\mathrm{LMH}}^{\mathrm{s}}\right]$ is equal to the identity matrix $\left[I_{n_{\mathrm{LM}}}\right]$ and therefore, it is a diagonal matrix, which is not the case, in general, for matrices $\left[D_{\mathrm{LMH}}^{\mathrm{s}}\right]$ and $\left[K_{\mathrm{LMH}}^{\mathrm{s}}\right]$.

\subsection{Numerical application for the multilevel reduced-order computational model}

In this section, the three frequency bounds $\omega_{\mathrm{L}}, \omega_{\mathrm{M}}$, and $\omega_{\mathrm{H}}$ are determined by analyzing the FRF for a given excitation (input) and for a set of observation points (outputs). The multilevel reduced-order computational model is constructed and validated by comparison with the classical reduced-order computational model.

\subsubsection{Description of the full-order computational model}

The full-order computational model is a three dimensional finite-element vibroacoustic model of an automobile. The finite element mesh of the structure and the acoustic cavity are shown in Fig. 2. There are $n_{\text {dof }}^{\mathrm{s}}+n_{\text {dof }}^{\mathrm{f}}=19984315$ degrees of freedom (structural displacements and acoustic pressure) in which $n_{\text {dof }}^{\text {fof }}$ is approximatively equal to 600000 .

An intensive computational effort has been carried out to compute the $n^{\mathrm{s}}=25685$ elastic modes (structure) and the $n^{\mathrm{f}}=4427$ acoustic modes (acoustic cavity) in the LF-MF-HF band, $[0,2000] \mathrm{Hz}$. These modes allow for constructing matrices $\left[\Phi^{\mathrm{s}}\right]$ and $\left[\Phi^{\mathrm{f}}\right]$ introduced in Sections 2.2 and 2.3 .

For calculating the structural and vibroacoustic frequency responses functions (FRF), the excitations (inputs) and the observations (outputs) are defined as follows. The structure excitations are the two points $c l v d$ and $c l v g$ located at engine supports. The structure observation points are two points on the floor ccuvg and ccurg, and again the points $c l v d$ and $c l v g$, as shown in Fig. 2 (top). The acoustic-cavity observation points are located at the points at ear height $a v d, a v g$, ard, and $\arg$ of passengers as shown in Fig. 2. (bottom).

\subsubsection{Frequency band limits $\omega_{\mathrm{L}}, \omega_{\mathrm{M}}$ and $\omega_{\mathrm{H}}$}

The frequency limits of the frequency bands (LF, MF, and HF) have been determined by analyzing the FRF for a given input (excitation at point $c l v g$ in $X$-direction) and 12 outputs 


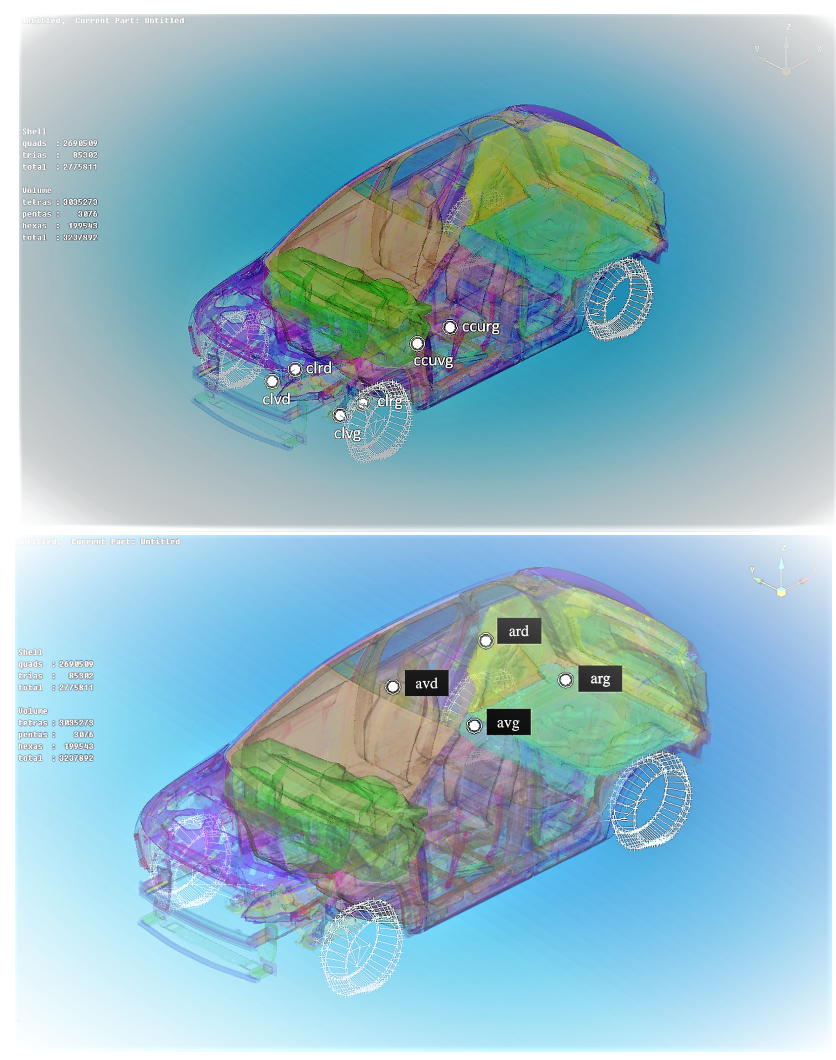

Figure 2: Finite element mesh of the structure and the acoustic cavity made up of 19984315 degrees of freedom (structural displacements and acoustic pressure). Top figure: structure excitation points (clvd and $c l v g$ ) and structure observation points (ccuvg, ccurg and again clvd, clvg). Bottom figure: acoustic-cavity observation points corresponding to four points at ear height (avd, $a v g$, ard, and $a r g$ ) of passengers. In the top figure, the labels can distinctly be seen by zooming.

(observations points ccuvg, ccurg, clvd, and $\operatorname{clvg}$ in $X-, Y$-, and Z-directions). Figures 3 to 6 show the graph of the mean value of these 12 FRFs with different frequency scaling. Comparing Figs. 3 and 4 allows for obtaining the value of the upper limit $\omega_{\mathrm{L}} / 2 \pi=200 \mathrm{~Hz}$ of the LF band. Comparing Figs. 3, 5, and 6 allows an estimation of the upper limit $\omega_{\mathrm{M}} / 2 \pi=800 \mathrm{~Hz}$ of the MF band, and of course, the upper limit of the validity domain of the reduced-order computational model, which is $\omega_{\mathrm{H}} / 2 \pi=2000 \mathrm{~Hz}$.

The determination of the value $d_{\mathrm{M}}$ (resp. $d_{\mathrm{L}}$ ) of polynomial parameter $d$ for the first (resp. second) spatial filtering depends on the elastic modes belonging to the MF (resp. LF) band. It is clear that the uncertainty on the values of $\omega_{\mathrm{M}}$ (resp. $\omega_{\mathrm{L}}$ ) yields uncertainties on the value of $d_{\mathrm{M}}\left(\right.$ resp. $d_{\mathrm{L}}$ ) as well on the construction of the multilevel reduced-order computational model. In the frequency band $\left[0, \omega_{\mathrm{M}}\right]$, there are $n_{\mathrm{LM}}=7470$ structural modes and $n_{\mathrm{LM}}^{\mathrm{f}}=419$ acoustic modes. 


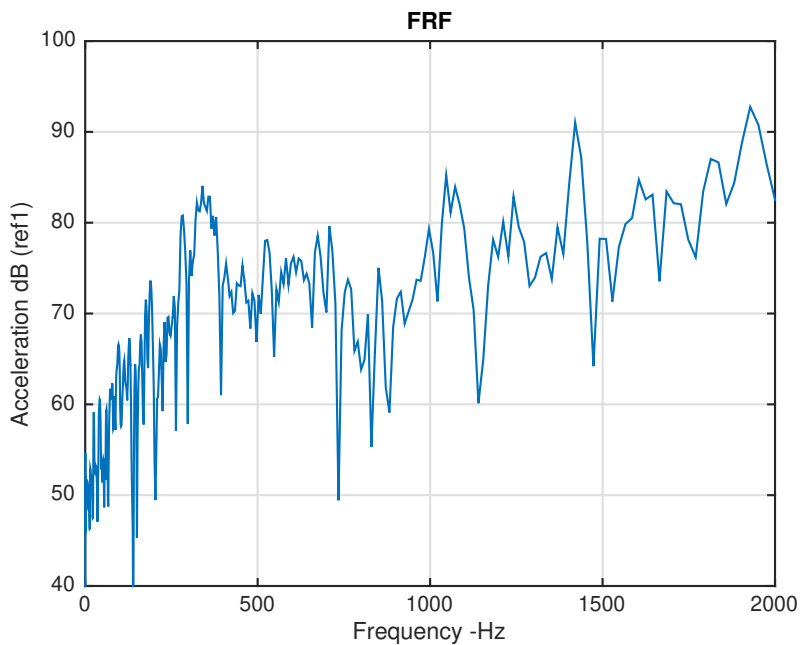

Figure 3: Graph of the mean value of the 12 FRFs for a same excitation at point clvg in $X$-direction and observation at points ccuvg, ccurg, clvd, and clvg in $X-, Y-$, and $Z$-directions. Vertical axis: acceleration in $\mathrm{dB}$. Horizontal axis: frequency $f=\omega / 2 \pi$ in $\mathrm{Hz}$ for $f \in$ $[0,2000] \mathrm{Hz}$.

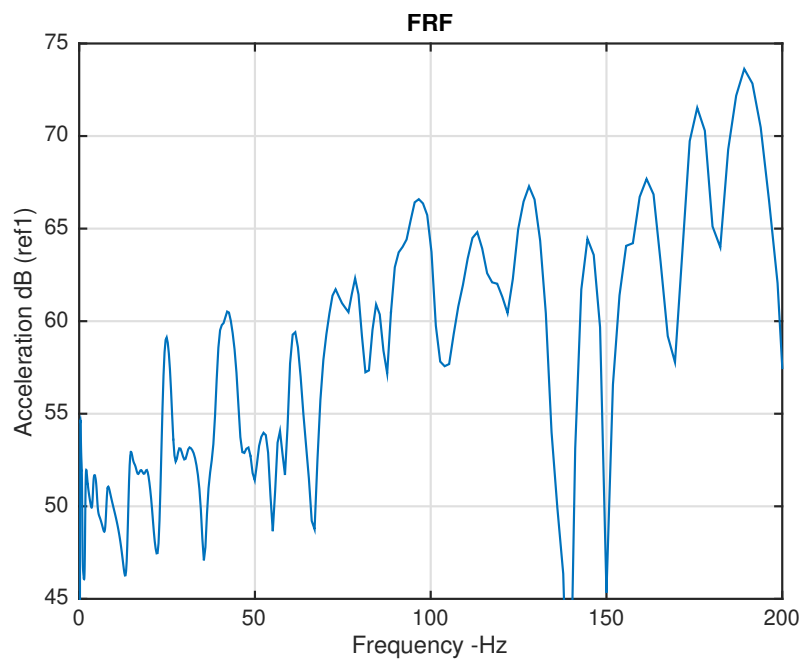

Figure 4: Graph of the mean value of the 12 FRFs for a same excitation at point clvg in $X$-direction and observation at points ccuvg, ccurg, clvd, and clvg in $X-, Y$-, and $Z$-directions. Vertical axis: acceleration in $\mathrm{dB}$. Horizontal axis: frequency $f=\omega / 2 \pi$ in $\mathrm{Hz}$ for $f \in[0,200] \mathrm{Hz}$.

\subsection{Complexity level for the HF band}

As presented in Section 4.2 , the complexity level of the HF band is quantified by the value $d_{\mathrm{H}}$ of polynomial degree parameter $d$ by studying the graph of mapping $d \mapsto \operatorname{conv}_{\mathrm{H}}(d)$ (see Fig. 7). It can be seen that $d_{\mathrm{H}}=32$. Nevertheless, it can also be seen that the values of $\operatorname{conv}_{\mathrm{H}}(d)$ are 


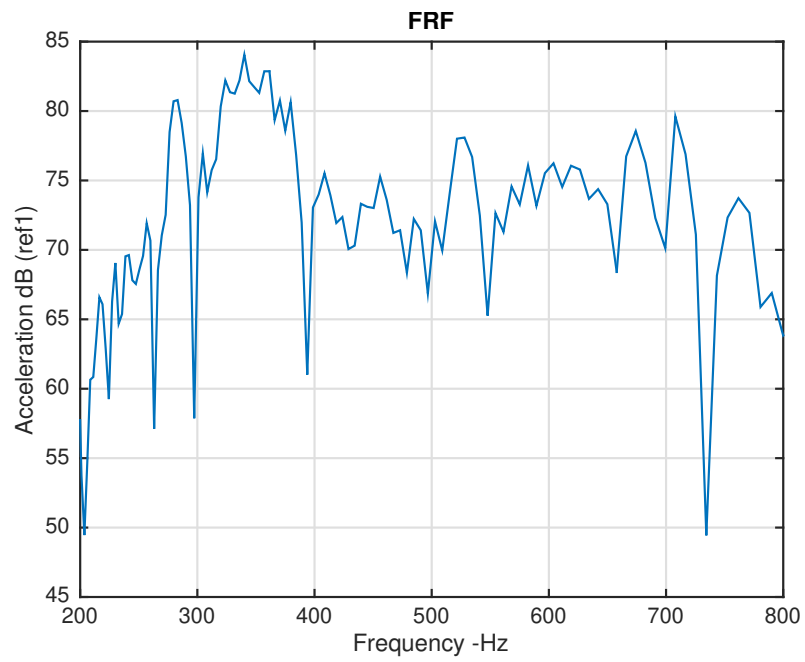

Figure 5: Graph of the mean value of the $12 \mathrm{FRFs}$ for a same excitation at point $c l v g$ in $X$-direction and observation at points ccuvg, ccurg, clvd, and $\operatorname{clvg}$ in $X-, Y-$, and $Z$-directions. Vertical axis: acceleration in $\mathrm{dB}$. Horizontal axis: frequency $f=\omega / 2 \pi$ in $\mathrm{Hz}$ for $f \in$ $[200,800] \mathrm{Hz}$.

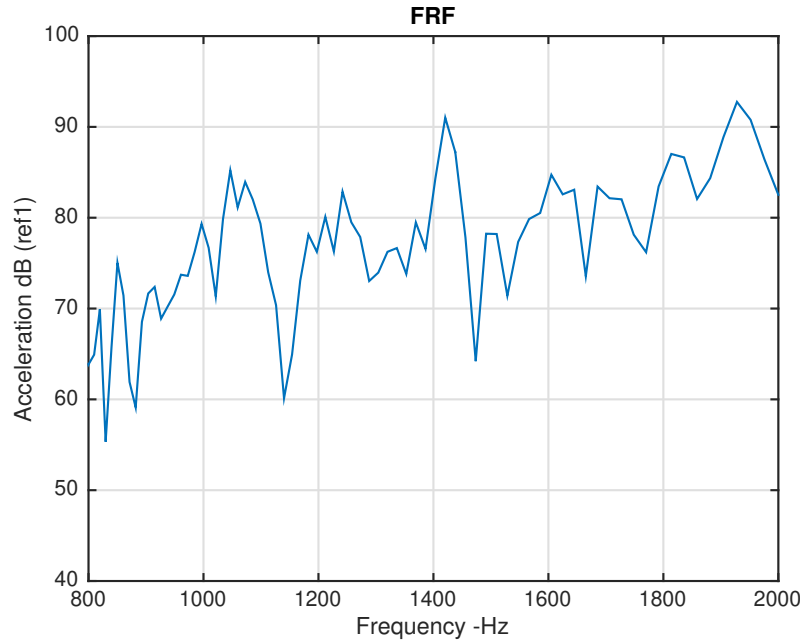

Figure 6: Graph of the mean value of the $12 \mathrm{FRFs}$ for a same excitation at point $c l v g$ in $X$-direction and observation at points ccuvg, ccurg, clvd, and $\operatorname{clvg}$ in $X-, Y$-, and $Z$-directions. Vertical axis: acceleration in $\mathrm{dB}$. Horizontal axis: frequency $f=\omega / 2 \pi$ in $\mathrm{Hz}$ for $f \in$ $[800,2000] \mathrm{Hz}$.

not negligible for small values of $d$. A possible interpretation is that there is no cutoff value $d_{\mathrm{H}}^{\text {cutoff }}$ of $d$, which can filter out all the elastic modes in the HF band into the columns of matrix [U $\left.\mathbb{U}^{\text {high }}\right]$. Consequently, the HF band cannot be considered as a purely high-complexity-level 
domain because a superposition of large structural displacements with very local displacements is observed. It is not expected that the values $d_{\mathrm{L}}$ and $d_{\mathrm{M}}$ be higher than $d_{\mathrm{H}}$ because it would mean

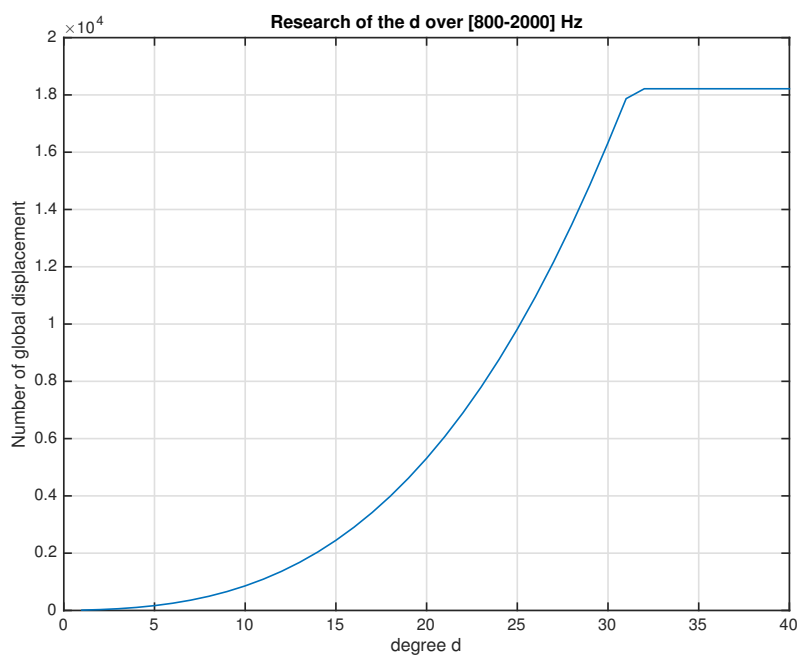

Figure 7: Graph of mapping $d \mapsto \operatorname{conv}_{\mathrm{H}}(d)$. Horizontal axis: polynomial degree $d$. Vertical axis: number of columns of matrix $\left[\mathbb{U}^{\text {high }}\right]$ for $[\mathbb{U}]=\left[\Phi_{\mathrm{H}}\right]$.

that the level of complexity of the LF or MF band is higher than the level of complexity of the HF band.

\subsection{Multilevel reduced-order model}

The value $\omega_{\mathrm{L}}$ is assumed to be equal to $200 \times 2 \pi \mathrm{rad} / \mathrm{s}$. The complexity level of the LF (resp. MF) band is quantified by the value $d_{\mathrm{L}}$ (resp. $d_{\mathrm{M}}$ ) of polynomial degree parameter $d$ by studying the graph of the mapping $d \mapsto \operatorname{conv}_{\mathrm{L}}(d)\left(\operatorname{resp} . d \mapsto \operatorname{conv}_{\mathrm{M}}(d)\right)$ that is shown in Fig. 8. It can be seen that $d_{\mathrm{M}}=22$ and $d_{\mathrm{L}}=14$ that are still lesser than $d_{\mathrm{H}}$ is in accordance with the usual expertise in structural dynamics for which the complexity level of the HF band is higher than the complexity level of the MF band, which is higher than the complexity level of the LF band. Again, there is no cutoff value $d_{\mathrm{M}}^{\text {cutoff }}$ of $d$, which can filter out all the elastic modes in the MF band into the columns of matrix [ $\left.\mathbb{U}^{\text {high }}\right]$. Consequently, the MF band cannot be considered as a purely (moderate) high-complexity-level domain only. Again, a superposition of large structural displacements with more or less local displacements can be observed. As a reminder, for constructing the multilevel reduced-order computational model, the parameters are $\omega_{\mathrm{L}}=200 / 2 \pi, \omega_{\mathrm{M}}=800 / 2 \pi, d_{\mathrm{L}}=14$ and $d_{\mathrm{M}}=22$. The number of columns of $\left[\mathbb{U}_{\mathrm{L}}\right]$ (resp. $\left[\mathbb{U}_{\mathrm{M}}\right]$ and $\left[\mathbb{U}_{\mathrm{H}}\right]$ ) is then $n_{\mathbb{U}_{\mathrm{L}}}=2040$ (resp. $n_{\mathbb{U}_{\mathrm{M}}}=4698$ and $n_{\mathbb{U}_{\mathrm{H}}}=742$ ). Three multilevel reduced-order computational models have been used by removing some columns of $\left[\mathbb{U}_{\mathrm{LMH}}\right]=$ $\left[\left[\mathbb{U}_{\mathrm{L}}\right]\left[\mathbb{U}_{\mathrm{M}}\right]\left[\mathbb{U}_{\mathrm{H}}\right]\right]$. Figure 9 (resp. Fig 10 ) presents the FRF for an excitation at point clvg in $X$-direction and an observation at point ccuvg (resp. ccurg) in $X$-direction for $\left[\mathbb{U}_{\mathrm{LMH}}\right]=$ $\left[\mathbb{U}_{\mathrm{M}}\right],\left[\mathbb{U}_{\mathrm{L}}\right],\left[\mathbb{U}_{\mathrm{H}}\right]$, and $\left[\mathbb{U}_{\mathrm{LMH}}\right]=\left[\left[\mathbb{U}_{\mathrm{L}}\right]\left[\mathbb{U}_{\mathrm{M}}\right]\left[\mathbb{U}_{\mathrm{H}}\right]\right]$ (black line). It has previously been checked that the FRF calculated with the multilevel reduced-order computational model with $\left[\mathbb{U}_{\mathrm{LMH}}\right]=$ $\left[\left[\mathbb{U}_{L}\right]\left[\mathbb{U}_{M}\right]\left[\mathbb{U}_{H}\right]\right]$ perfectly fits the FRF calculated by the ROM defined by Eqs. (5) and (6). These results allow us to conclude that, in general, $\left[\mathbb{U}_{\mathrm{M}}\right]$ does not span the same vector space as 

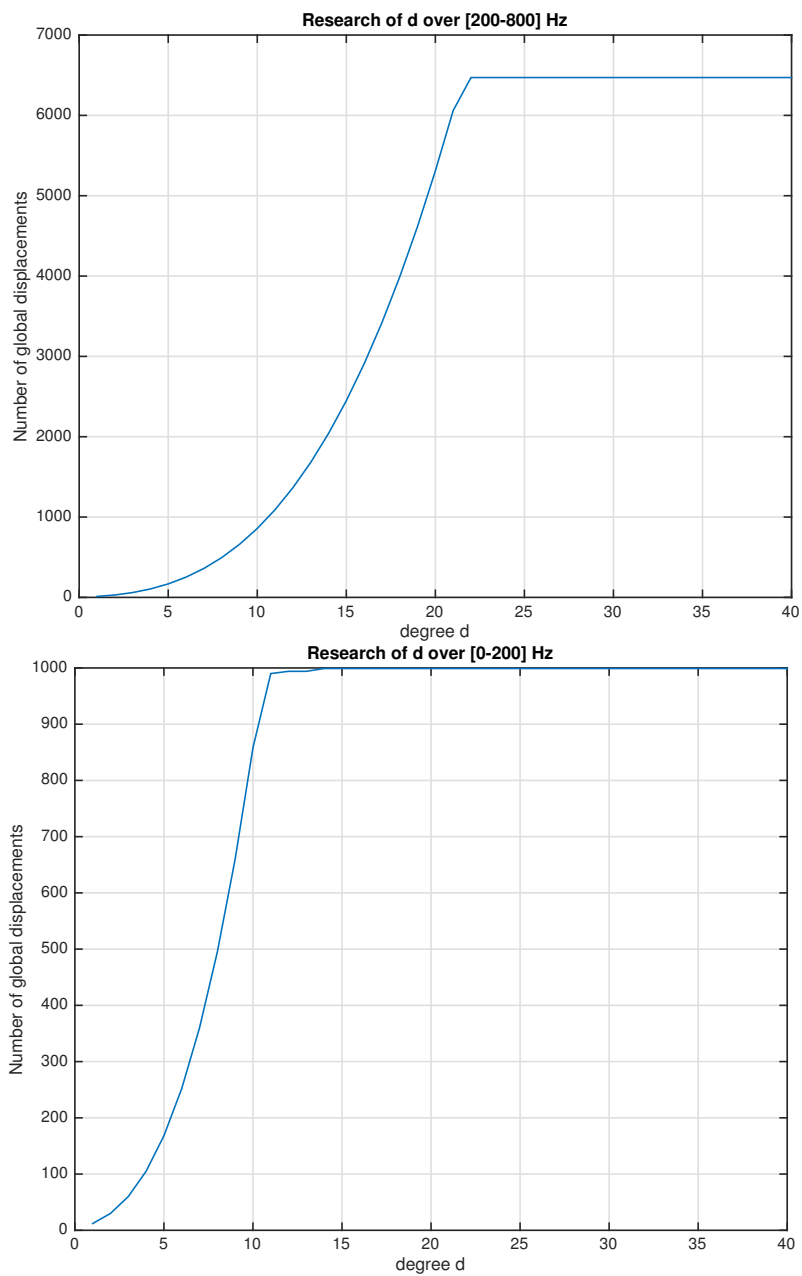

Figure 8: Complexity level in the LH and MF bands. Top figure: graph of $d \mapsto \operatorname{conv}_{\mathrm{M}}(d)$. Bottom figure: graph of $d \mapsto \operatorname{conv}_{\mathrm{L}}(d)$. Horizontal axis: polynomial degree $d$. Vertical axis: Number of columns of matrix $\left[\mathbb{U}^{\text {low }}\right]$ for $[\mathbb{U}]=\left[\Phi_{M}\right]$ (top) and for $[\mathbb{U}]=\left[\Phi_{M}\right]$ (bottom).

the elastic modes in the MF band (note that we have $n_{\mathrm{LM}} \nsucc n_{\mathbb{U}_{\mathrm{M}}}$ ). Figure 11 presents the FRF for an excitation at point $c l v g$ in $X$-direction and four observations of the acoustic pressure (in $\mathrm{dB}$ ) at points avg, arg, avd, and ard for $\left[\mathbb{U}_{\mathrm{LMH}}\right]=\left[\mathbb{U}_{\mathrm{M}}\right],\left[\mathbb{U}_{\mathrm{L}}\right],\left[\mathbb{U}_{\mathrm{H}}\right]$, and $\left[\mathbb{U}_{\mathrm{LMH}}\right]=\left[\left[\mathbb{U}_{\mathrm{L}}\right]\left[\mathbb{U}_{\mathrm{M}}\right]\left[\mathbb{U}_{\mathrm{H}}\right]\right]$. As already explained, it has previously been checked that this FRF computed with the multilevel reduced-order computational model with $\left[\mathbb{U}_{\mathrm{LMH}}\right]=\left[\left[\mathbb{U}_{\mathrm{L}}\right]\left[\mathbb{U}_{\mathrm{M}}\right]\left[\mathbb{U}_{\mathrm{H}}\right]\right]$ perfectly fits the FRF computed by the ROM defined by Eqs. (5) and (6). In Fig. 11] it can be seen that none of the bases $\left[\mathbb{U}_{L}\right],\left[\mathbb{U}_{M}\right]$, and $\left[\mathbb{U}_{H}\right]$ (for representing displacement vector $u(\omega)$ ) is able to predict alone the responses for such a complex vibroacoustic system. 


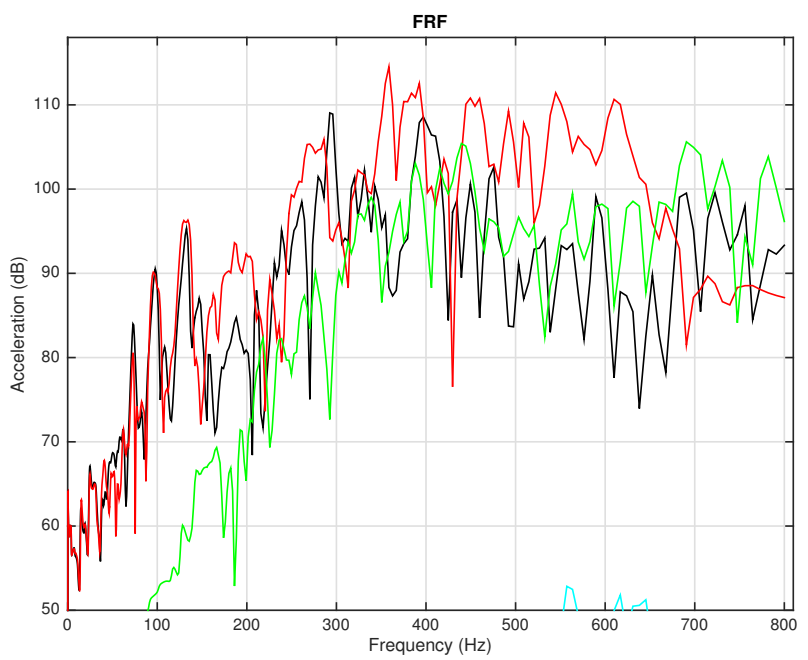

Figure 9: FRF for an excitation at point clvg and an observation at point ccuvg, both in $X$-direction, for $\left[\mathbb{U}_{\mathrm{LMH}}\right]=\left[\mathbb{U}_{\mathrm{M}}\right]($ green line $),=\left[\mathbb{U}_{\mathrm{L}}\right]($ red line $),=\left[\mathbb{U}_{\mathrm{H}}\right]($ cyan line, but out of range in the figure), and $\left[\mathbb{L}_{\mathrm{LMH}}\right]=\left[\left[\mathbb{U}_{\mathrm{L}}\right]\left[\mathbb{U}_{\mathrm{M}}\right]\left[\mathbb{U}_{\mathrm{H}}\right]\right]$ (black line). Horizontal axis: frequency $\omega / 2 \pi$ in Hz. Vertical axis: acceleration in $\mathrm{dB}$.

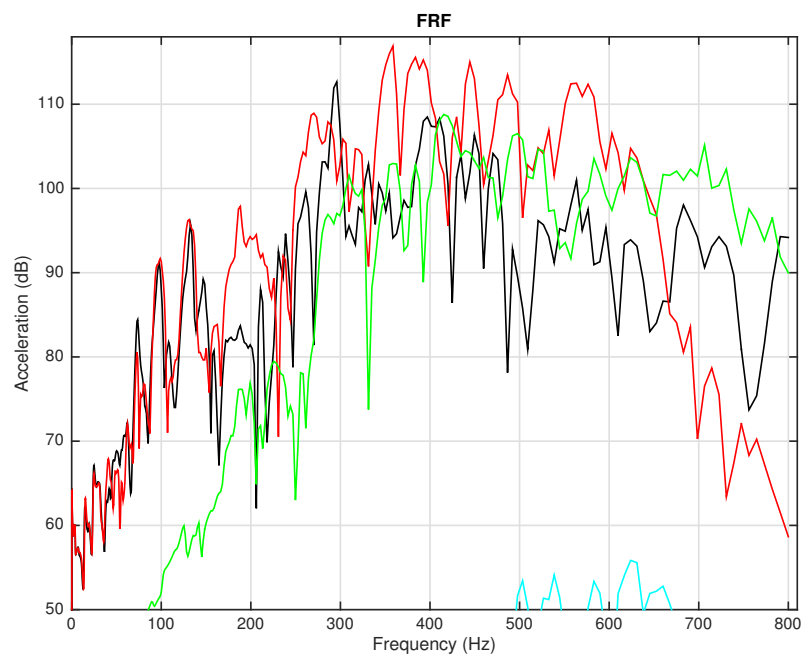

Figure 10: FRF for an excitation at point $c l v g$ and an observation at point ccurg, both in $X$-direction, for $\left[\mathbb{U}_{\mathrm{LMH}}\right]=\left[\mathbb{U}_{\mathrm{M}}\right]$ (green line), $=\left[\mathbb{U}_{\mathrm{L}}\right]($ red line $),=\left[\mathbb{U}_{\mathrm{H}}\right]$ (cyan line, but out of range in the figure), and $\left[\mathbb{U}_{\mathrm{LMH}}\right]=\left[\left[\mathbb{U}_{\mathrm{L}}\right]\left[\mathbb{U}_{\mathrm{M}}\right]\left[\mathbb{U}_{\mathrm{H}}\right]\right]$ (black line). Horizontal axis: frequency $\omega / 2 \pi$ in Hz. Vertical axis: Acceleration in dB.

\section{Stochastic multilevel reduced-order computational model in vibroacoustics}

The objective of this section is to take into account the model uncertainties induced by the modeling errors by using the nonparametric probabilistic approach and to construct the stochastic 

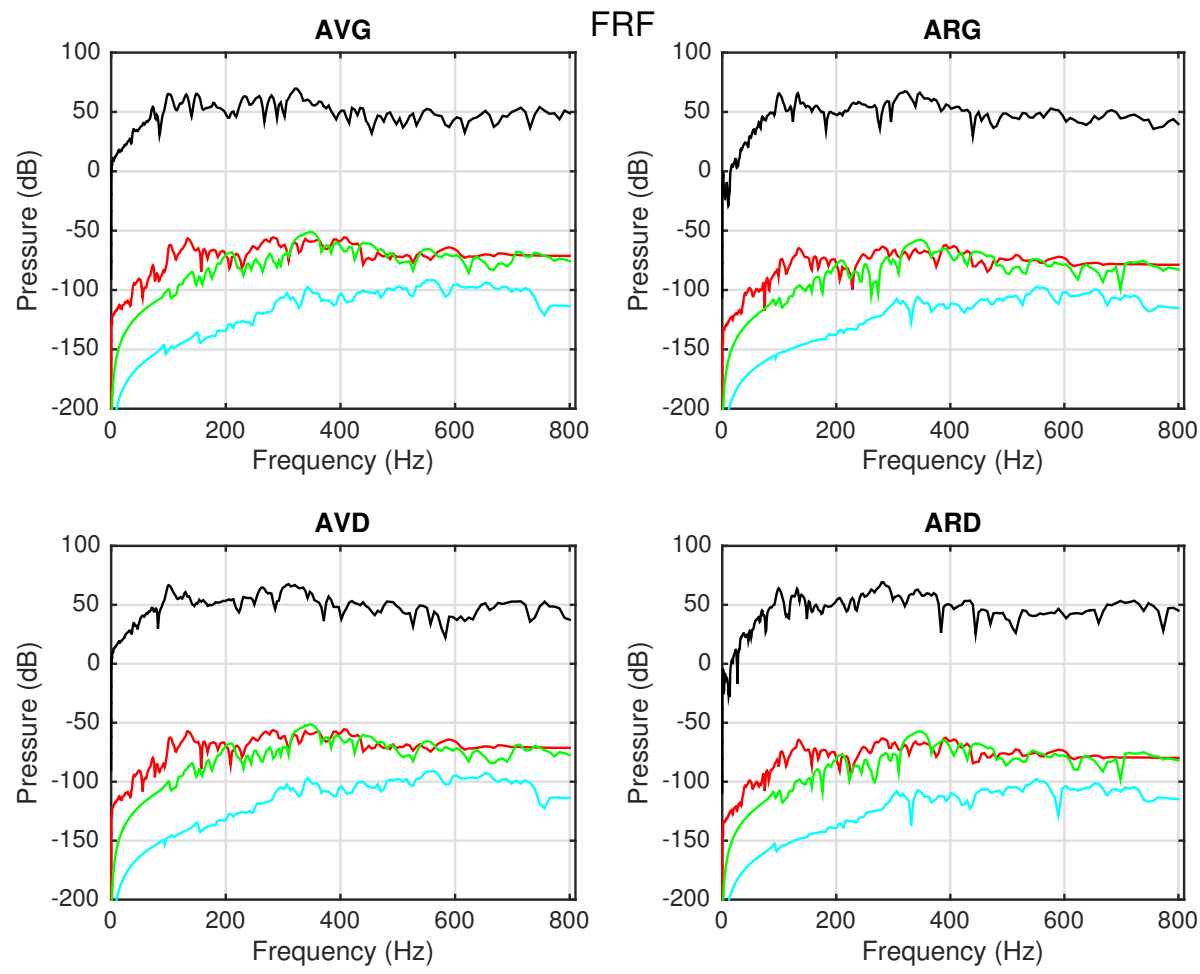

Figure 11: FRF for an excitation at point $c l v g$ in $X$-direction and four observations of acoustic pressure (in $\mathrm{dB}$ ) at points avg (top left), arg (top right), avd (bottom left), and ard (bottom right) for $\left[\mathbb{U}_{\mathrm{LMH}}\right]=\left[\mathbb{U}_{\mathrm{M}}\right]($ green line $),=\left[\mathbb{U}_{\mathrm{L}}\right]$ (red line), $=\left[\mathbb{U}_{\mathrm{H}}\right]$ (cyan line, but out of range in the figure), and $\left[\mathbb{U}_{\mathrm{LMH}}\right]=\left[\left[\mathbb{U}_{\mathrm{L}}\right]\left[\mathbb{U}_{\mathrm{M}}\right]\left[\mathbb{U}_{\mathrm{H}}\right]\right]$ (black line). Horizontal axis: frequency $\omega / 2 \pi \mathrm{in} \mathrm{Hz}$. Vertical axis: acoustic pressure in $\mathrm{dB}$.

multilevel reduced-order computational vibroacoustic model. The nonparametric probabilistic approach for the model uncertainties (see [64, 42]) consists in directly substituting the matrices of the multilevel reduced-order computational model presented in Section 4.4 by random matrices. The probabilistic model of these random matrices has been constructed by the use of the MaxEnt Principle. The hyperparameters of the probabilistic model for each random matrix consist of the mean value of the random matrix and a dispersion coefficient that controls the level of statistical fluctuations, and which reflects the level of uncertainties. We start by introducing the nonparametric probabilistic approach in the classical stochastic reduced-order computational model and then its implementation in the multilevel reduced-order computational model is presented.

\subsection{Nonparametric probabilistic approach of uncertainties for the classical reduced-order com- putational model}

It is assumed that there are no model uncertainties in the acoustic cavity but only on the structure and on its coupling with the acoustic cavity. The classical stochastic reduced-order computational model is then obtained by substituting $\left[\mathcal{C}_{\mathrm{LM}}\right],\left[\mathcal{M}_{\mathrm{LM}}^{\mathrm{s}}\right],\left[\mathcal{D}_{\mathrm{LM}}^{\mathrm{s}}\right],\left[\mathcal{K}_{\mathrm{LM}}^{\mathrm{s}}\right]$ in Eqs. (7) 
and $(8)$ by random matrices $\left[\mathcal{C}_{\mathrm{LM}}\right],\left[\mathcal{M}_{\mathrm{LM}}^{\mathrm{s}}\right],\left[\mathcal{D}_{\mathrm{LM}}^{\mathrm{s}}\right],\left[\mathcal{K}_{\mathrm{LM}}^{\mathrm{s}}\right]$, which yields

$$
\begin{gathered}
\left(-\omega^{2}\left[\mathcal{M}_{\mathrm{LM}}^{\mathrm{s}}\right]+i \omega\left[\mathcal{D}_{\mathrm{LM}}^{\mathrm{s}}\right]+\left[\mathcal{K}_{\mathrm{LM}}^{\mathrm{s}}\right]\right) \mathbf{Q}^{\mathrm{s}}(\omega)+\left[\mathcal{C}_{\mathrm{LM}}\right] \mathbf{Q}^{\mathrm{f}}(\omega)=\mathbb{E}_{\mathrm{LM}}^{\mathrm{s}}(\omega), \\
\left(-\omega^{2}\left[\mathcal{M}^{\mathrm{f}}\right]+i \omega\left[\mathcal{D}^{\mathrm{f}}\right]+\left[\mathcal{K}^{\mathrm{f}}\right]\right) \mathbf{Q}^{\mathrm{f}}(\omega)+\omega^{2}\left[\mathcal{C}_{\mathrm{LM}}\right]^{T} \mathbf{Q}^{\mathrm{s}}(\omega)=\mathbf{0},
\end{gathered}
$$

in which $\mathbf{Q}^{\mathrm{s}}(\omega)\left(\right.$ resp. $\left.\mathbf{Q}^{\mathrm{f}}(\omega)\right)$ is a $\mathbb{C}^{n^{\mathrm{s}}}$ (resp. $\left.\mathbb{C}^{n^{\mathrm{f}}}\right)$-valued random vector. The displacement vector $u(\omega)$ (resp. the acoustic pressure vector $\mathbb{p}(\omega))$ is then replaced by the $\mathbb{C}_{\text {dof }- \text { valued random vector }}^{n^{s}}$ $\mathbf{U}(\omega)$ (resp. the $\mathbb{C}_{\text {dof }}^{\mathrm{f}}$-valued random vector $\mathbf{P}(\omega)$ ) such that

$$
\mathbf{U}(\omega)=\left[\Phi_{\mathrm{LM}}^{\mathrm{s}}\right] \mathbf{Q}^{\mathrm{s}}(\omega) \quad, \quad \mathbf{P}(\omega)=\left[\Phi^{\mathrm{f}}\right] \mathbf{Q}^{\mathrm{f}}(\omega) .
$$

Random matrix $\left[\mathcal{C}_{\mathrm{LM}}\right]$ belongs to the ensemble $\mathrm{SE}^{\text {rect }}$ (see [42]) with a dispersion coefficient $\delta_{\mathcal{C}_{\mathrm{LM}}}$ and with a mean value $\left[\mathcal{C}_{\mathrm{LM}}\right]$. Random matrix $\left[\mathcal{M}_{\mathrm{LM}}^{\mathrm{s}}\right]$ (resp. $\left[\mathcal{D}_{\mathrm{LM}}^{\mathrm{s}}\right]$ and $\left[\mathcal{K}_{\mathrm{LM}}^{\mathrm{s}}\right]$ ) belongs to the ensemble $\mathrm{SE}^{+}$(see [42]) with dispersion coefficient $\delta_{\mathcal{M}_{\mathrm{LM}}^{\mathrm{s}}}\left(\right.$ resp. $\delta_{\mathcal{D}_{\mathrm{LM}}^{\mathrm{s}}}$ and $\delta_{\mathcal{K}_{\mathrm{LM}}^{\mathrm{s}}}$ ) and with a mean value $\left[\mathcal{M}_{\mathrm{LM}}^{\mathrm{s}}\right]$ (resp. $\left[\mathcal{D}_{\mathrm{LM}}^{\mathrm{s}}\right]$, and $\left.\left[\mathcal{K}_{\mathrm{LM}}^{\mathrm{s}}\right]\right)$.

\subsection{Nonparametric probabilistic approach of uncertainties for the multilevel reduced-order computational model}

Again, it is assumed that there are no model uncertainties in the acoustic cavity but only on the structure and on its coupling with the acoustic cavity. The stochastic multilevel reducedorder computational model is constructed by substituting $\left[C_{\mathrm{LMH}}\right],\left[M_{\mathrm{LMH}}^{\mathrm{s}}\right],\left[D_{\mathrm{LMH}}^{\mathrm{s}}\right]$, and $\left[K_{\mathrm{LMH}}^{\mathrm{s}}\right]$ in Eqs. 41 and 42 by random matrices $\left[\mathbf{C}_{\mathrm{LMH}}\right],\left[\mathbf{M}_{\mathrm{LMH}}^{\mathrm{s}}\right],\left[\mathbf{D}_{\mathrm{LMH}}^{\mathrm{s}}\right]$, and $\left[\mathbf{K}_{\mathrm{LMH}}^{\mathrm{s}}\right]$. We then obtain

$$
\begin{gathered}
\left(-\omega^{2}\left[\mathbf{M}_{\mathrm{LMH}}^{\mathrm{s}}\right]+i \omega\left[\mathbf{D}_{\mathrm{LMH}}^{\mathrm{s}}\right]+\left[\mathbf{K}_{\mathrm{LMH}}^{\mathrm{s}}\right]\right) \mathbf{Q}_{\mathrm{LMH}}^{\mathrm{s}}(\omega)+\left[\mathbf{C}_{\mathrm{LMH}}\right] \mathbf{Q}^{\mathrm{f}}(\omega)=\mathbb{L}_{\mathrm{LMH}}^{\mathrm{s}}(\omega), \\
\left(-\omega^{2}\left[M^{\mathrm{f}}\right]+i \omega\left[D^{\mathrm{f}}\right]+\left[K^{\mathrm{f}}\right]\right) \mathbf{Q}^{\mathrm{f}}(\omega)+\omega^{2}\left[\mathbf{C}_{\mathrm{LMH}}\right]^{T} \mathbf{Q}_{\mathrm{LMH}}^{\mathrm{s}}(\omega)=\mathbf{0},
\end{gathered}
$$

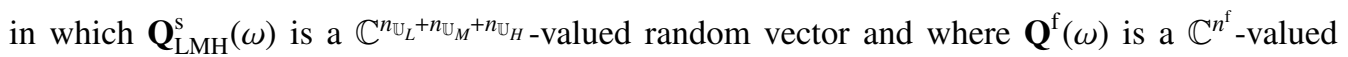
random vector. The displacement vector $u(\omega)$ (resp. the acoustic pressure vector $p(\omega))$ is then

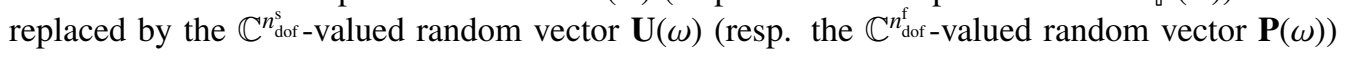
such that

$$
\mathbf{U}(\omega)=\left[\mathbb{U}_{\mathrm{LMH}}\right] \mathbf{Q}_{\mathrm{LMH}}^{\mathrm{s}}(\omega), \mathbf{P}(\omega)=\left[\Phi^{\mathrm{f}}\right] \mathbf{Q}^{\mathrm{f}}(\omega) .
$$

Concerning the probabilistic models of $\left[\mathbf{C}_{\mathrm{LMH}}\right],\left[\mathbf{M}_{\mathrm{LMH}}^{\mathrm{s}}\right],\left[\mathbf{D}_{\mathrm{LMH}}^{\mathrm{s}}\right]$, and $\left[\mathbf{K}_{\mathrm{LMH}}^{\mathrm{s}}\right]$, a naive approach would consist in substituting matrices $\left[\mathcal{C}_{\mathrm{LM}}\right],\left[\mathcal{M}_{\mathrm{LM}}^{\mathrm{s}}\right],\left[\mathcal{D}_{\mathrm{LM}}^{\mathrm{s}}\right]$, and $\left[\mathcal{K}_{\mathrm{LM}}^{\mathrm{s}}\right]$ in the expressions of matrices $\left[C_{\mathrm{LMH}}\right],\left[M_{\mathrm{LMH}}^{\mathrm{s}}\right],\left[D_{\mathrm{LMH}}^{\mathrm{s}}\right]$, and $\left[K_{\mathrm{LMH}}^{\mathrm{s}}\right]$ given by Eqs. (43) to (46) by the random matrices $\left[\mathcal{C}_{\mathrm{LM}}\right],\left[\mathcal{M}_{\mathrm{LM}}^{\mathrm{s}}\right],\left[\mathcal{D}_{\mathrm{LM}}^{\mathrm{s}}\right]$, and $\left[\mathcal{K}_{\mathrm{LM}}^{\mathrm{s}}\right]$ that have been introduced in Section 5.1. However, such a probabilistic model would exactly be the same as the one presented in Section 5.1 and consequently, would not be interesting. Hence, the probabilistic model of random matrix $\left[\mathbf{C}_{\mathrm{LMH}}\right]$ is constructed by substituting matrix $\left[\mathcal{C}_{\mathrm{LM}}\right]$ in Eq. 46 by random matrix $\left[\mathcal{C}_{\mathrm{LM}}\right]$ that has been introduced in Section 5.1. We then have

$$
\left[\mathbf{C}_{\mathrm{LMH}}\right]=\left[\mathbb{Q}_{\mathrm{LMH}}\right]^{T}\left[\mathcal{C}_{\mathrm{LM}}\right] .
$$

Furthermore, let $[\mathbf{A}]$ be any of random matrices $\left[\mathbf{M}_{\mathrm{LMH}}^{\mathrm{s}}\right],\left[\mathbf{D}_{\mathrm{LMH}}^{\mathrm{s}}\right]$, and $\left[\mathbf{K}_{\mathrm{LMH}}^{\mathrm{s}}\right]$ for which the mean values are $\left[M_{\mathrm{LMH}}^{\mathrm{s}}\right]=E\left\{\left[\mathbf{M}_{\mathrm{LMH}}^{\mathrm{s}}\right]\right\},\left[D_{\mathrm{LMH}}^{\mathrm{s}}\right]=E\left\{\left[\mathbf{D}_{\mathrm{LMH}}^{\mathrm{s}}\right]\right\}$, and $\left[K_{\mathrm{LMH}}^{\mathrm{s}}\right]=E\left\{\left[\mathbf{K}_{\mathrm{LMH}}^{\mathrm{s}}\right]\right\}$ with $E\{\cdot\}$ is the mathematical expectation operator. The Cholesky factorization of matrix $[A]=E\{[\mathbf{A}]\}$ is written as $[A]=\left[L_{A}\right]^{T}\left[L_{A}\right]$ in which $\left[L_{A}\right]$ is an upper triangular matrix. The probabilistic model 
of random matrix $[\mathbf{A}]$ is then written as

$$
[\mathbf{A}]=\left[L_{A}\right]^{T}\left[\mathbf{G}_{A}\right]\left[L_{A}\right],
$$

where the random matrix $\left[\mathbf{G}_{A}\right]$ is written as

$$
\left[\mathbf{G}_{\mathrm{A}}\right]=\left[\begin{array}{ccc}
{\left[\mathbf{G}_{\mathrm{L}, A}\right]} & {[0]} & {[0]} \\
{[0]} & {\left[\mathbf{G}_{\mathrm{M}, A}\right]} & {[0]} \\
{[0]} & {[0]} & {\left[\mathbf{G}_{\mathrm{H}, A}\right.}
\end{array}\right]
$$

In Eq. [56, the random matrices $\left[\mathbf{G}_{\mathrm{L}, A}\right],\left[\mathbf{G}_{\mathrm{M}, A}\right]$, and $\left[\mathbf{G}_{\mathrm{H}, A}\right]$ belong to ensemble $\mathrm{SG}_{0}^{+}$(see [42]) with dimension $n_{\mathbb{U}_{\mathrm{L}}} \times n_{\mathbb{U}_{\mathrm{L}}}, n_{\mathbb{U}_{\mathrm{M}}} \times n_{\mathbb{U}_{\mathrm{M}}}$, and $n_{\mathbb{U}_{\mathrm{H}}} \times n_{\mathbb{U}_{\mathrm{H}}}$, and with dispersion coefficients $\delta_{\mathrm{L}, A}, \delta_{\mathrm{M}, A}$, and $\delta_{\mathrm{H}, A}$. Consequently, the construction of the stochastic multilevel reduced-order computational model involves ten dispersion coefficients that are

$\delta_{\mathcal{C}_{\mathrm{LMH}}}$ for $\left[\mathbf{C}_{\mathrm{LMH}}\right]$,

$\delta_{\mathrm{L}, \mathbf{M}_{\mathrm{LMH}}^{\mathrm{s}}}^{\mathrm{s}}, \delta_{\mathrm{M}, \mathbf{M}_{\mathrm{LMH}}^{\mathrm{s}}}, \delta_{\mathrm{H}, \mathbf{M}_{\mathrm{LMH}}^{\mathrm{s}}}$ for $\left[\mathbf{M}_{\mathrm{LMH}}^{\mathrm{s}}\right]$,

$\delta_{\mathrm{L}, \mathbf{D}_{\mathrm{LMH}}^{\mathrm{s}}}, \delta_{\mathrm{M}, \mathbf{D}_{\mathrm{LMH}}^{\mathrm{s}}}$ and $\delta_{\mathrm{H}, \mathbf{D}_{\mathrm{LMH}}^{\mathrm{s}}}$ for $\left[\mathbf{D}_{\mathrm{LMH}}^{\mathrm{s}}\right]$,

$\delta_{\mathrm{L}, \mathbf{K}_{\mathrm{LMH}}^{\mathrm{s}}}, \delta_{\mathrm{M}, \mathbf{K}_{\mathrm{LMH}}^{\mathrm{s}}}, \delta_{\mathrm{H}, \mathbf{K}_{\mathrm{LMH}}^{\mathrm{s}}}$ for $\left[\mathbf{K}_{\mathrm{LMH}}^{\mathrm{s}}\right]$.

\subsection{Numerical results obtained with classical stochastic reduced-order computational model}

The Monte-Carlo numerical method is used for solving Eqs. (48) to (50) in order to quantify the role played by uncertainties in the FRF. The number of realizations is 46 and there are 200 frequency points distributed in $\log _{10}$-scale between $20 \mathrm{HZ}$ and $800 \mathrm{~Hz}$. The relatively small value of the number of realizations has been chosen to make calculations feasible with acceptable CPU time using the available computers. It was verified that this value made it possible to qualitatively and quantitatively preserve the prediction of the upper envelopes of the confidence domains. The main influence of this small number of realizations is a relatively poor estimate of the lower envelopes of the confidence domains. These lower envelopes would be smoother with a greater number of realizations, but the upper envelopes would remain stable as we have been able to numerically verify by choosing a lower number of realizations.

Figure 12 shows the random structure-structure FRF for the structural excitation at point $c l v d$ in $X$-direction and for the structural observation at point ccuvg in $X$-direction, with $\delta_{\mathcal{C}_{\mathrm{LM}}}=0.4$, $\delta_{\mathcal{M}_{\mathrm{LM}}^{\mathrm{s}}}=\delta_{\mathcal{D}_{\mathrm{LM}}^{\mathrm{s}}}=\delta_{\mathcal{K}_{\mathrm{LM}}^{\mathrm{s}}}=0.3$.

Figure 13 shows the random structure-acoustic FRF for the structural excitation at point $c l v g$ in $X$-direction and for the acoustical observation at point at ear height $a v g$, with $\delta_{\mathcal{C}_{\mathrm{LM}}}=0.4$, $\delta_{\mathcal{M}_{\mathrm{LM}}^{\mathrm{s}}}=\delta_{\mathcal{D}_{\mathrm{LM}}^{\mathrm{s}}}=\delta_{\mathcal{K}_{\mathrm{LM}}^{\mathrm{s}}}=0.3$.

\subsection{Numerical results obtained with stochastic multilevel reduced-order computational model}

Similarly to the classical stochastic reduced-order computational model, the Monte-Carlo numerical method is used for solving Eqs. (51) to (53). The number of realizations is always 46 with 200 frequency points distributed in $\log _{10}$-scale between $20 \mathrm{HZ}$ and $800 \mathrm{~Hz}$.

Two FRFs are analyzed. The first one, referred as EX1, is the structure-structure FRF for which the structural excitation is at point $c l v d$ and the structural observation is at point $c c u v g$, both in $X$-direction. The second one, referred as EX2, is the structure-acoustic FRF for which the structural excitation is at point $c l v g$ in $X$-direction and the acoustical observation is at point at ear height $a v g$. 


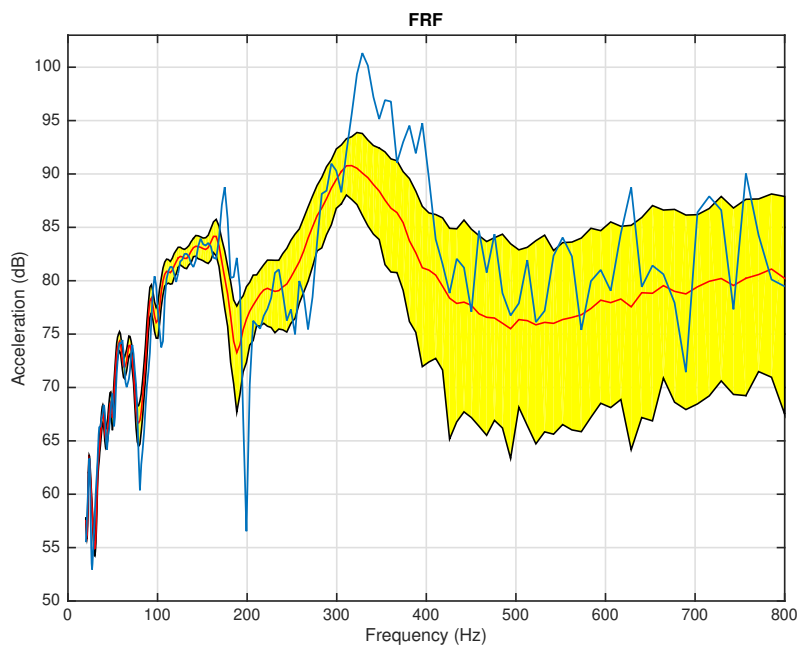

Figure 12: Structure-structure FRF estimated with the classical stochastic ROM for the structural excitation at point $c l v d$ and observation $c c u v g$, both in $X$-direction. Confidence region at of $95 \%$ (yellow), nominal (blue), statistical mean value (red). Horizontal axis: frequency in Hz. Vertical axis: acceleration in $\mathrm{dB}$.

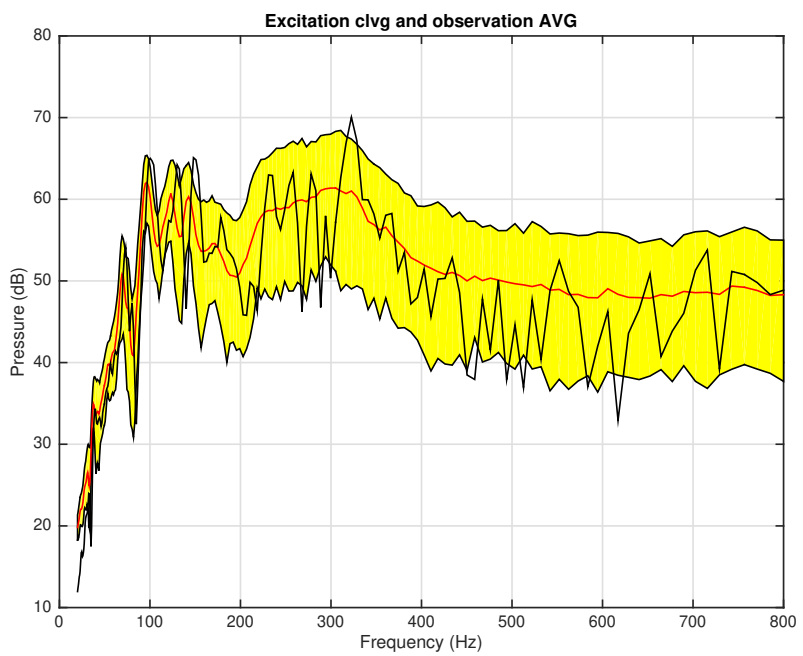

Figure 13: Structure-acoustic FRF estimated with the classical stochastic ROM for the structural excitation at point clvg in $X$-direction and for the acoustical observation at point avg. Confidence region at $95 \%$ (yellow), nominal (black), and statistical mean value (red). Horizontal axis: frequency in Hz. Vertical axis: acoustic pressure in $\mathrm{dB}$.

The calculations have been carried out for 4 sets of dispersion coefficients values. These sets are labelled as cases (i), (ii), (ii), and (iv) and are defined in Table 1 For these 4 cases, $\delta_{\mathcal{C}_{\mathrm{LMH}}}=0.4$. The results are presented in Figs. 14 to 21 In each figure, it can be seen the confidence region for a probability level of $95 \%$, the statistical mean value, and the nominal FRF 
calculated with the deterministic computational vibroacoustic model.

Table 1: For $L, M$, and $H$, values of the dispersion coefficients $\delta_{\mathcal{M}_{\mathrm{LMH}}^{\mathrm{s}}}, \delta_{\mathcal{D}_{\mathrm{LMH}}^{\mathrm{s}}}$, and $\delta_{\mathcal{D}_{\mathrm{LMH}}^{\mathrm{s}}}$, for the 4 cases (i), (ii), (iii), and (iv)

\begin{tabular}{|c|c|c|c|c|}
\hline & (i) & (ii) & (iii) & (iv) \\
\hline$\delta_{\mathrm{L}, \mathcal{M}_{\mathrm{LMH}}^{\mathrm{s}}}$ & 0.3 & 0.05 & 0.05 & 0.3 \\
$\delta_{\mathrm{L}, \mathcal{D}_{\mathrm{LMH}}^{\mathrm{s}}}$ & 0.3 & 0.05 & 0.05 & 0.3 \\
$\delta_{\mathrm{L}, \mathcal{K}_{\mathrm{LMH}}^{\mathrm{s}}}$ & 0.25 & 0.05 & 0.05 & 0.25 \\
\hline$\delta_{\mathrm{M}, \mathcal{M}_{\mathrm{LMH}}^{\mathrm{s}}}^{\mathrm{s}}$ & 0.05 & 0.3 & 0.05 & 0.3 \\
$\delta_{\mathrm{M}, \mathcal{D}_{\mathrm{LMH}}^{\mathrm{s}}}$ & 0.05 & 0.3 & 0.05 & 0.3 \\
$\delta_{\mathrm{M}, \mathcal{K}_{\mathrm{LMH}}^{\mathrm{s}}}$ & 0.05 & 0.25 & 0.05 & 0.25 \\
\hline$\delta_{\mathrm{H}, \mathcal{M}_{\mathrm{LMH}}^{\mathrm{s}}}$ & 0.05 & 0.05 & 0.3 & 0.3 \\
$\delta_{\mathrm{H}, \mathcal{D}_{\mathrm{LMH}}^{\mathrm{s}}}$ & 0.05 & 0.05 & 0.3 & 0.3 \\
$\delta_{\mathrm{H}, \mathcal{K}_{\mathrm{LMH}}^{\mathrm{s}}}$ & 0.05 & 0.05 & 0.25 & 0.25 \\
\hline
\end{tabular}

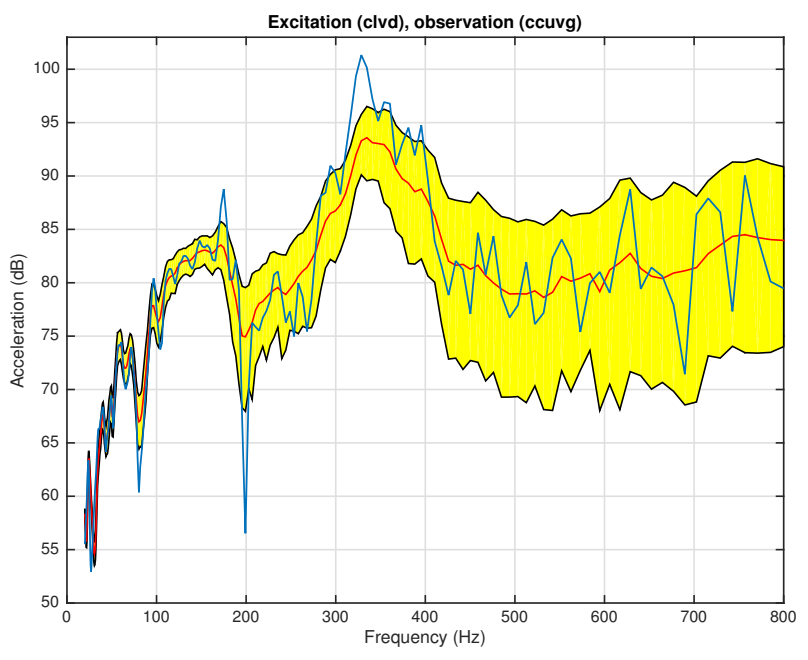

Figure 14: Structure-structure FRF (EX1) for case (i): confidence region at 95\% (yellow), nominal (blue), and statistical mean (red).

\subsection{Discussions}

The nominal FRFs are calculated by using the deterministic computational vibroacoustic model. At a given frequency, the value of a nominal FRF may or may not belong to the confidence region calculated with the classical stochastic reduced-order computational model or with the stochastic multilevel reduced-order computational model. The nominal value is not a reference as would be experimental measurements. Unfortunately, experimental measurements are not available for this vibroacoustic system. Consequently, the identification of the dispersion coefficients by solving statistical inverse problems and experiments cannot be done. This is the 


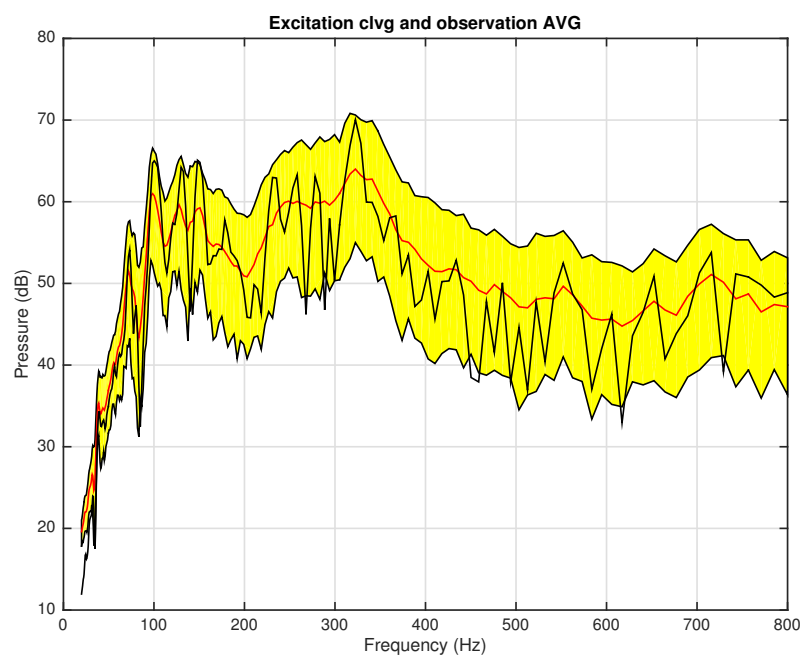

Figure 15: Structure-acoustic FRF (EX2) for case (i): confidence region at 95\% (yellow), nominal (black), and statistical mean (red).

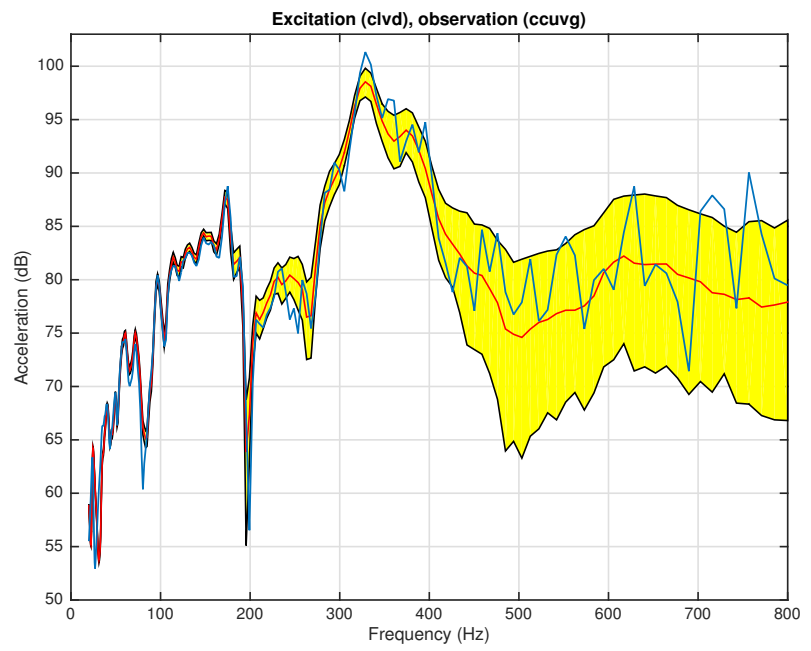

Figure 16: Structure-structure FRF (EX1) for case (ii): confidence region at 95\% (yellow), nominal (blue), and statistical mean (red).

reason why a sensitivity analysis with respect to the level of uncertainties that are controlled by the dispersion coefficients have been carried out.

The introduction of model uncertainties, which makes it possible to take into account the modeling errors, is precisely done to give robustness to the results given by the computational model. There is no reason the responses of the nominal deterministic system always belong to the confidence regions.

The Nastran software has been used with available computation servers, to compute the elastic modes, the acoustic modes, and to export the generalized matrices of the vibroacoustic system, 


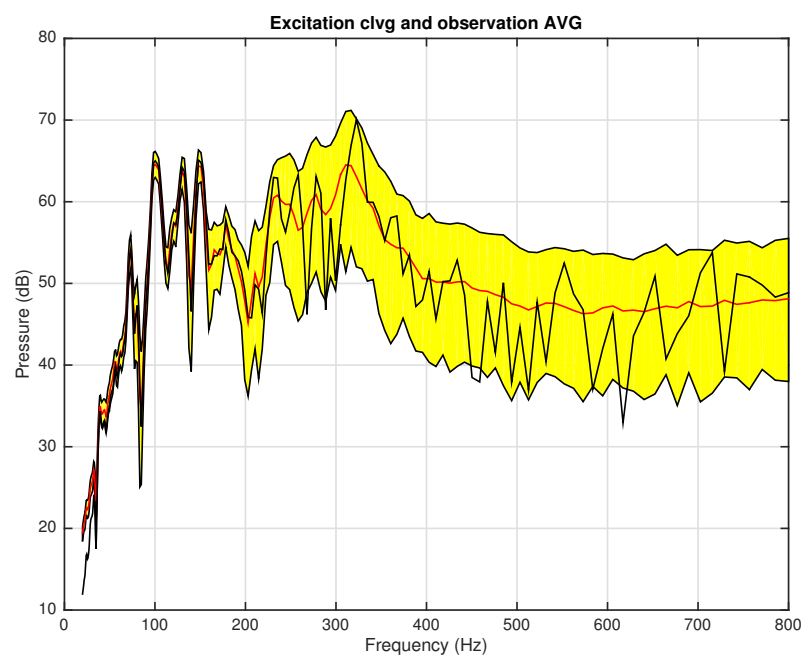

Figure 17: Structure-acoustic FRF (EX2) for case (ii): confidence region at 95\% (yellow), nominal (black), and statistical mean (red).

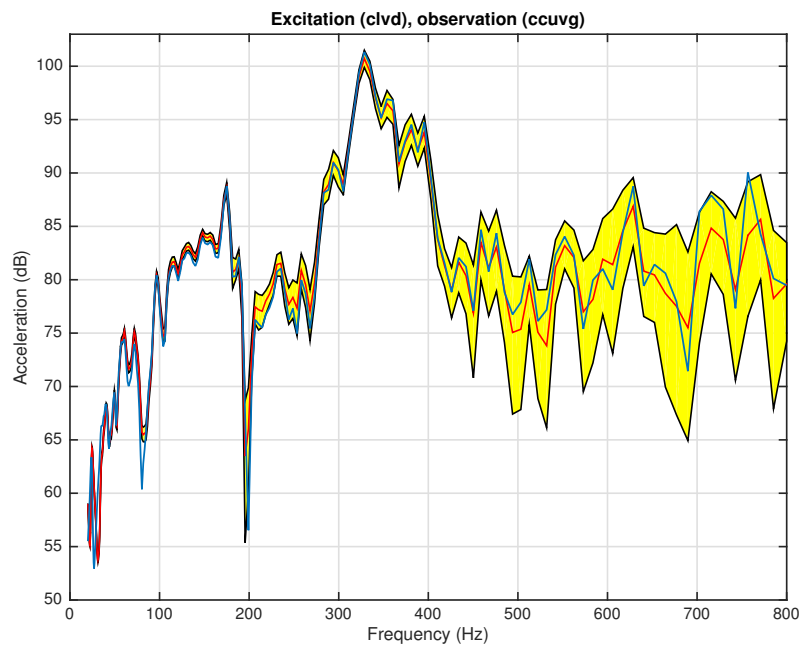

Figure 18: Structure-structure FRF (EX1) for case (iii): confidence region at 95\% (yellow), nominal (blue), and statistical mean (red).

in particular the generalized full coupling matrix between the structure and the acoustic cavity. Taking into account the very large dimension of the computational vibroacoustic model (approximately 20 million degrees of freedom), it has not been possible to do the computations in one run, for all the frequency band of analysis (which would have made it possible to export the generalized full matrix of coupling), but had to be made by frequency sub-band. In this case, for a given frequency sub-band, Nastran generates only the diagonal block of the generalized coupling matrix for the elastic modes and the acoustic modes that belong to this sub-band. Thus, it is not possible to obtain the full generalized coupling matrix for the entire band of analysis, but only 


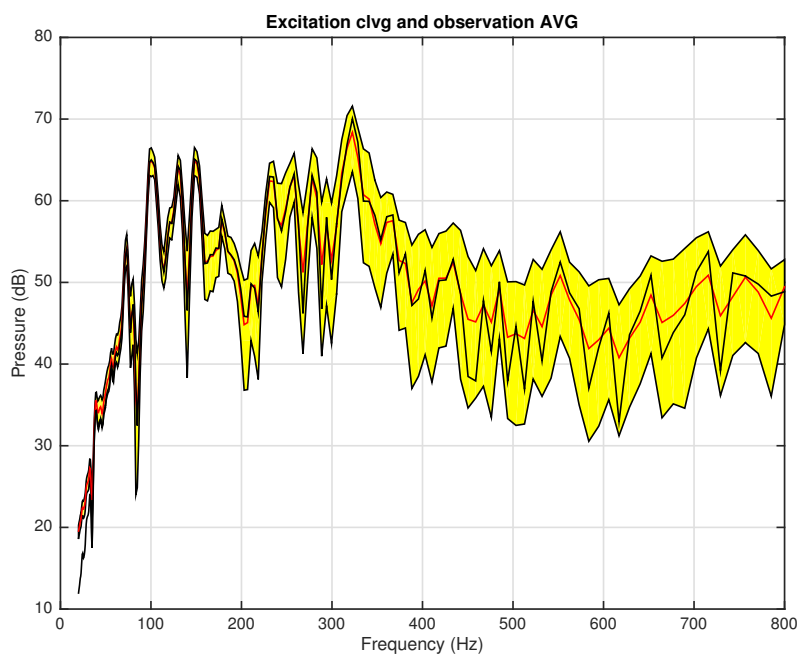

Figure 19: Structure-acoustic FRF (EX2) for case (iii): confidence region at 95\% (yellow), nominal (black), and statistical mean (red).

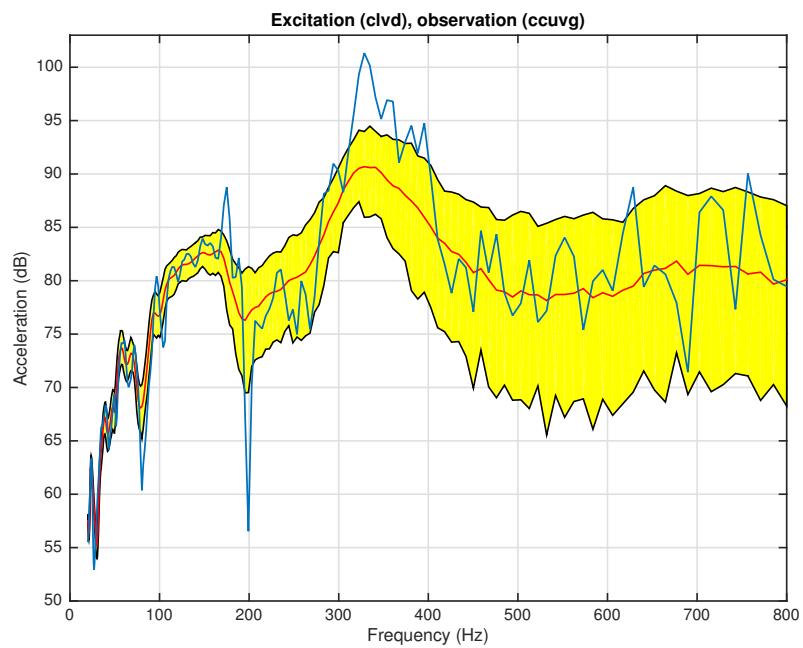

Figure 20: Structure-structure FRF (EX1) for case (iv): confidence region at 95\% (yellow), nominal (blue), and statistical mean (red).

the diagonal blocks, the extra-diagonal blocks then being zero, which is not perfectly correct. Under these conditions, the prediction of the acoustic response of the vibroacoustic system is an approximation whose level of approximation cannot be evaluated given the size of the problem.

On the other hand, to test the methodology and algorithms, the nonparametric probabilistic model of uncertainties has been implemented in a general framework, which means that the random matrix germs are full as the theory of random matrices specifies for the ensembles of random matrices considered. However, as it has just been explained, the generalized coupling matrix of the nominal model, which should be full (taking into account all the couplings between the 


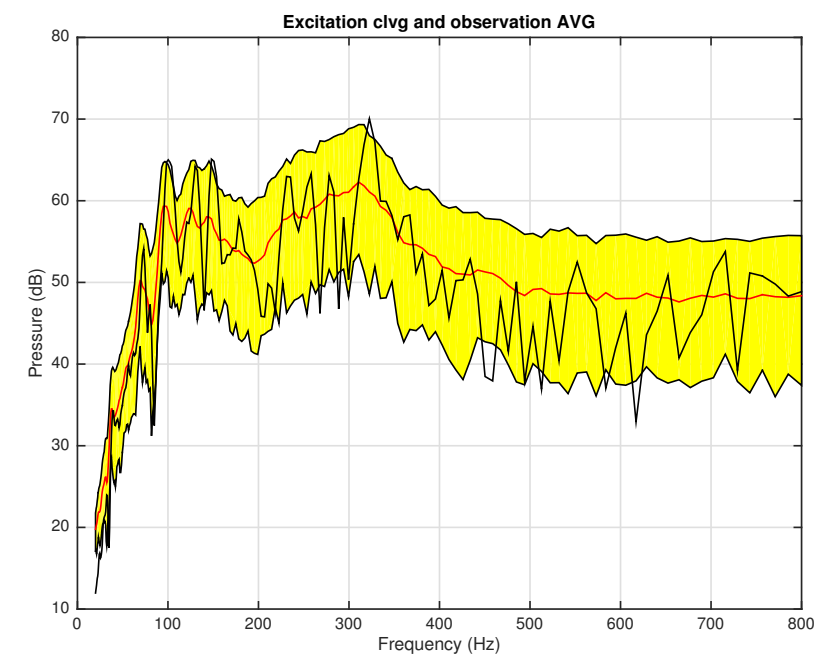

Figure 21: Structure-acoustic FRF (EX2) for case (iv): confidence region at 95\% (yellow), nominal (black), and statistical mean (red).

elastic and acoustic modes), is not and presents extra-diagonal blocks, which are zero matrices. The construction of the random matrices associated with this generalized coupling matrix of the nominal model, therefore substitutes the zero blocks of this nominal matrix by non-zero random blocks. Therefore, for the prediction of acoustic responses, the level of approximation used, between the nominal vibroacoustic model and the classical or multilevel stochastic computational model, is not exactly the same. This could induce an additional small error in the interpretation of the acoustic results of the vibroacoustic system when comparing the acoustic response of the nominal system with the acoustic response of the classical or multilevel stochastic model. Despite these approximations, the errors induced remain low and do not modify quantitatively the results presented, both for the structure-structure FRF and for structure-acoustic FRF. In accordance with what it is indicated as perspectives, a necessary development would be to obtain the complete vibroacoustic coupling matrix and to see what is its influence on the vibroacoustic FRF. It should be noted that such a development is directly linked to the possibilities of the commercial software, here Nastran, used and not to the developments, strictly speaking, of the proposed method.

In the light of the comments, which have been made above, the results obtained are consistent and are the expected results. The stochastic multilevel ROM takes better into account the uncertainties as a function of the LF and MF frequency bands than the classical stochastic ROM. Examination of all the Figures (Figs. 12 to 21 ) clearly shows that, a same and unique analysis can be given for the structure-structure FRF and the structure-acoustic FRF. From now on, we will no longer differentiate between these two types of FRF.

Four different sets (cases (i) to (iv)) of dispersion coefficients (that is to say of uncertainty levels) are used for the three complexity levels L, M, and H (see Section 4). A comment is formulated hereinafter for each one of these sets.

The first set that features high-level uncertainties for the L complexity level and low-level 
uncertainties for the $\mathrm{M}$ and $\mathrm{H}$ complexity levels can be seen in Figs. 14 and 15 .

The results for the second set that presents a high level of uncertainty for the M complexity level and a low level of uncertainty for the $\mathrm{L}$ and $\mathrm{H}$ complexity levels, are given in Figs. 16 and 17. This case gives narrow confidence regions in the LF band, whose width increases with the frequency.

For the third set, which deals with a high level of uncertainty for the $\mathrm{H}$ complexity level and a low level of uncertainty for the $\mathrm{L}$ and $\mathrm{M}$ complexity levels, the results are shown in Figs. 18 and 19. The width of confidence region as well as the position of the statistical mean yield a robust model. This case of the uncertainty level yields a very thin confidence region that grows with the frequency.

The last set introduces a high level of uncertainty for the three levels of complexity, L, M, and $\mathrm{H}$, and the results are shown in Figs. 20 and 21 .

Comparing these results with the results given by the classical stochastic ROM, it can be concluded that the classical stochastic ROM is a special case of the stochastic multilevel ROM and that the stochastic multilevel ROM is better than the classical stochastic ROM. First of all the robustness with regard to uncertainty level does not depend solely on the choice of the polynomial degrees but also of the three complexity levels. Finally, it is well known that the MF band is sensitive to uncertainties unlike the LF band that has very little sensitivity to model uncertainties. In the multilevel method, it is therefore consistent to consider a significant level of uncertainties for the M complexity level and a very low level of uncertainty for the L complexity level. This situation corresponds to the second set (case (ii)) of values for the dispersion coefficients for which the results are given in Figs. 16 and 17. We obtain a good result in terms of taking into account the uncertainties as a function of the frequency and which is consistent with the fact that the value of $\omega_{L} / 2 \pi=200 \mathrm{~Hz}$ gives a good separation of the 3 complexity levels: $\mathrm{L}, \mathrm{M}$, and $\mathrm{H}$.

\section{Conclusions and perspectives}

This work has proposed an improvement of the general method of spatial filtering previously developed for complex structures and has presented an extension to vibroacoustic systems made up of a complex structure coupled with an internal acoustic cavity. The systems studied are all the more complex as the number of degrees of freedom increases with the frequency band of analysis. The modified method has been made to construct a multi-complexity-level displacement basis in order to obtain the multilevel ROM.

The principal use is for robust analysis of complex vibroacoustic systems over a broad frequency band for which the probabilistic model of uncertainties induced by modeling errors is adapted to each one of the three vibration regimes, LF, MF, and HF. For that, a multilevel stochastic ROM has been developed for the structure, which is able to take into account the variability induced by the overlap in the three vibration regimes.

This work is in continuation of the work in [80, 81] for which a complete reformulation of the method has been proposed in this paper, for which a novel presentation and numerical developments are performed, and for which novel interpretations are given. For large scale computational vibroacoustic model, we have constructed a predictive stochastic multilevel ROM whose dimension is inferior to the usual ROM constructed by using the classical modal analysis. Algorithms have specifically been developed to be used for very large computational models without encountering problems related to the limitation of Random Acces Memory (RAM) and with numerical 
costs that remain low. This capability of the proposed method has required in-depth methodological and algorithmic reflection. In particular, the numerical analysis and the developed algorithm have been written to improve the existing codes. The high dimension of the computational model compelled us to propose an efficient computation to optimize time calculation (CPU) and data storage limitations (RAM). The database (modal analysis, lumped mass matrix, vibroacoustic modal coupling matrix) has been computed from a dedicated software (Nastran). All the proposed approach and the post-processing have been implemented in Matlab. Consequently, the approach proposed is nonintrusive with respect to commercial software. We have automated some parameters like the range of the first eigenvalue problem and not use the cutoff frequency in the process of the filtering.

The applications have been performed for an automobile whose computational vibroacoustic model is made up an acoustic cavity (cockpit) of 600000 dofs coupled with a complex structure (car) (of nearly 19 million of dofs). Several cases have been considered in order to test the method, the numerical analysis, and the algorithms. The validation has been given and the capability of the proposed approach has been tested, for the stochastic multilevel reduced-order computational vibroacoustic model. This nonparametric stochastic multilevel ROM gives better results than the classical nonparametric stochastic ROM with respect to the taking into account of uncertainties as a function of the frequency.

\section{References}

[1] J. Durand, C. Soize, L. Gagliardini, Structural-acoustic modeling of automotive vehicles in presence of uncertainties and experimental identification and validation, The Journal of the Acoustical Society of America 123(3) (2008) 1513-1525. doi:10.1121/1.2953316

[2] A. Arnoux, A. Batou, C. Soize, L. Gagliardini, Stochastic reduced order computational model of structures having numerous local elastic modes in low frequency dynamics, Journal of Sound and Vibration 332(16) (2013) 36673680. doi:10.1016/j.jsv.2013.02.019

[3] A. Arnoux, C. Soize, L. Gagliardini, Reduced-order computational model for low-frequency dynamics of automobiles, Advances in Mechanical Engineering 31036 (2013) 1-12. doi:10.1155/2013/310362

[4] L. Gagliardini, Dispersed vibroacoustic responses of industrial products: What are we able to predict?, The 26th International Conference on Noise and Vibration engineering (ISMA2014), Leuven, 15-17 September 2003-011555 (2014) 17-37.

[5] I. Bucher, S. Braun, Left eigenvectors: Extraction from measurements and physical interpretation, Journal of Applied Mechanics ASME 64(1) (1997) 97-105.

[6] P. Hansen, The truncated svd as a method for regularization, BIT Numerical Mathematics 27 (4) (1987) $534-553$.

[7] R. Guyan, Reduction of stiffness and mass matrices, AIAA Journal 3(2) (1965) 380-380.

[8] R. Guyan, A method for selecting master dof in dynamic substructuring using the guyan condensation method, Computers \& Structures 45(5-6) (1992) 941-946.

[9] R. Guyan, Flexural wave-propagation behavior of lumped mass approximations, Computers \& Structures 12(6) (1980) 805-812.

[10] H. Chan, C. Cai, Y. Cheung, High convergence order finite elements with lumped mass matrix, Journal of Sound and Vibration 165(2) (1993) 193-207.

[11] M. Jensen, Convergence studies of dynamic analysis by using the finite element method with lumped mass matrix, International Journal for Numerical Methods in Engineering 139(11) (1996) 1879-1888.

[12] R. Langley, P. Bremner, A hybrid method for the vibration analysis of complex structural-acoustic systems, The Journal of the Acoustical Society of America 105(3) (1999) 1657-1671.

[13] L. Ji, B. Mace, R. Pinnington, A mode-based approach for the mid-frequency vibration analysis of coupled longand short-wavelength structures, Journal of Sound and Vibration 289(1-2) (2006) 148-170.

[14] Y. Hahn, N. Kikuchi, Identification of global modeshape from a few nodal eigenvectors using simple free-form deformation, Engineering with Computers 21 (2) (2005) 115-128.

[15] J. Guyader, Characterization and reduction of dynamic models of vibrating systems with high modal density, Journal of Sound and Vibration 328(4-5) (2009) 488-506.

[16] J. Guyader, Modal sampling method for the vibration study of systems of high modal density, The Journal of the Acoustical Society of Americam 88(5) (1990) 2269-2276. 
[17] A. Noor, M. Anderson, W. Greene, Continuum models for beam- and platelike-lattice structures, AIAA Journal 16(12) (1978) 1219-1228.

[18] J. Planchard, Vibrations of nuclear fuel assemblies: a simplified model, Nuclear Engineering and Design 86 (3) (1985) 383-391.

[19] J. Sigrits, D. Broc, Dynamic analysis of a tube bundle with fluid-structure interaction modelling using a homogenisation method, Computer Methods in Applied Mechanics and Engineering 197(9-12) (2008) 1080-1099.

[20] R. Craig, A Review of time domain and frequency domain component mode synthesis method in combined experimental-analytical modeling of dynamic structural systems, D.R. Martinez and A.K. Miller, New York, 1985.

[21] D. de Klerk, D. Rixen, S. Voormeeren, General framework for dynamic substructuring: History, review, and classification of techniques, AIAA Journal 4 (2008) 1169-1181.

[22] A. Leung, Dynamic Stiffness and Substructures, Springer-Verlag, Berlin, 1993.

[23] R. Ohayon, C. Soize, R. Sampaio, Variational-based reduced-order model in dynamic substructuring of coupled structures through a dissipative physical interface: Recent advances, Archives of Computational Methods in Engineering 21 (3) (2014) 321-329. doi:10.1007/s11831-014-9107-y

[24] J. Argyris, S. Kelsey, The analysis of fuselages of arbitrary cross-section and taper: A DSIR sponsored research program on the development and application of the matrix force method and the digital computer, Aircraft Engineering and Aerospace Technology 31(3) (1959) 62-74.

[25] J. Przemieniecki, Matrix structural analysis of substructures, AIAA Journal 1(1) (1963) 138-147.

[26] B. Irons, Structural eigenvalue problems - elimination of unwanted variables, AIAA Journal 3(5) (1965) 961-962.

[27] W. Hurty, Vibrations of structural systems by component mode synthesis, Journal of Engineering Mechanics ASCE 86(4) (1960) 51-70.

[28] W. Hurty, Dynamic analysis of structural systems using component modes, AIAA Journal 3(4) (1965) 678-685.

[29] R. Craig, M. Bampton, Coupling of substructures for dynamic analyses, AIAA Journal 6(7) (1968) $1313-1319$.

[30] K. Bathe, S. Gracewski, On nonlinear dynamic analysis using substructuring and mode superposition, Computers \& Structures 13(5) (1981) 699-707.

[31] C. Farhat, M. Geradin, On a component mode synthesis method and its application to incompatible substructures, Computers \& Structures 51(5) (1994) 459-473.

[32] L. Meirovitch, A. Hale, On the substructure synthesis method, AIAA Journal 19(7) (1981) 940-947.

[33] L. Meirovitch, M. Kwak, Rayleigh-ritz based substructure synthesis for flexible multibody systems, AIAA Journal 29(10) (1991) 1709-1719.

[34] S. Voormeeren, P. van der Valk, D. Rixen, Generalized methodology for assembly and reduction of component models for dynamic substructuring, AIAA Journal 49(5) (2011) 1010-1020.

[35] S. Voormeeren, P. van der Valk, D. Rixen, Vibration analysis of structures by component mode substitution, AIAA Journal 9(7) (1971) 1255-1261.

[36] R. Mac Neal, Vibration analysis of structures by component mode substitution, Computers \& Structures 1(4) (1971) 581-601.

[37] S. Rubin, Improved component-mode representation for structural dynamic analysis, AIAA Journal 13(8) (1975) 995-1006.

[38] D. Markovic, K. Park, A. Ibrahimbegovic, Reduction of substructural interface degrees of freedom in flexibilitybased component mode synthesis, International Journal for Numerical Methods in Engineering 70(2) (2007) 163180.

[39] R. Ohayon, R. Sampaio, C. Soize, Dynamic substructuring of damped structures using singular value decomposition, Journal of Applied Mechanics ASME 64(2) (1997) 292-298. doi:10.1115/1.2787306

[40] K. Park, Y. Park, Dynamic substructuring of damped structures using singular value decomposition, AIAA Journal 42(6) (2004) 1236-1245.

[41] D. Rixen, A dual Craig-Bampton method for dynamic substructuring, Journal of Computational and Applied Mathematics 168 (1-2) (2004) 383-391.

[42] C. Soize, Uncertainty Quantification. An Accelerated Course with Advanced Applications in Computational Engineering, Springer, New York, 2017. doi:10.1007/978-3-319-54339-0

[43] R. Ibrahim, Parametric Random Vibration, John Wiley and Sons, New York, 1985.

[44] J. Beck, L. Katafygiotis, Updating models and their uncertainties - i: Bayesian statistical framework, Journal of Engineering Mechanics ASCE 124(4) (1998) 455-461.

[45] R. Mace, W. Worden, G. Manson, Uncertainty in structural dynamics, Journal of Sound and Vibration 288(3) (2005) 431-790.

[46] G. Schuëller, H. Pradlwarter, Uncertain linear systems in dynamics: Retrospective and recent developments by stochastic approaches, Engineering Structures 31 (11) (2009) 2507-2517.

[47] C. Soize, Stochastic modeling of uncertainties in computational structural dynamics - recent theoretical advances, Journal of Sound and Vibration 332(10) (2013) 2379-2395. doi:10.1016/j • jsv.2011.10.010

[48] G. Schuëller, Computational methods in stochastic mechanics and reliability analysis, Computer Methods in Ap- 
plied Mechanics and Engineering 194(12-16) (2005) 1251-1795.

[49] G. Schuëller, Uncertainties in structural mechanics and analysis - computational methods, Computer \& Structures 83(14) (2005) 1031-1150.

[50] G. Schuëller, Developments in stochastic structural mechanics, Archive of Applied Mechanics 75 (10-12) (2006) $755-773$.

[51] G. Deodatis, P. Spanos, Computational stochastic mechanics, Probabilistic Engineering Mechanics 23(2-3) (2008) $103-346$.

[52] R. Ghanem, Stochastic Finite Elements: A Spectral Approach, Revised edition, Dover Publications, New York, 1991.

[53] C. Soize, R. Ghanem, Physical systems with random uncertainties: chaos representations with arbitrary probability measure, SIAM Journal on Scientific Computing 26 (2) (2004) 395-410. doi : 10.1137/S1064827503424505

[54] O. Le Maitre, O. Knio, Spectral Methods for Uncertainty Quantification with Applications to Computational Fluid Dynamics, Springer, Heidelberg, 2010.

[55] R. Ghanem, D. Higdon, H. Owhadi, Handbook of Uncertainty Quantification, Springer International Publishing Switzerland, 2017.

[56] T. Bui-Thanh, K. Willcox, O. Ghattas, Parametric reduced-order models for probabilistic analysis of unsteady aerodynamic applications, AIAA Journal 46(10) (2008) 2520-2529.

[57] J. Degroote, J. Vierendeels, K. Willcox, Interpolation among reduced-order matrices to obtain parameterized models for design, optimization and probabilistic analysis, International Journal for Numerical Methods in Engineering 63(2) (2010) 207-230.

[58] Y. Marzouk, H. Najm, L. Rahn, Stochastic spectral methods for efficient bayesian solution of inverse problems, Journal of Computational Physics 224 (2) (2007) 560-586.

[59] D. Galbally, K. Fidkowski, K. Willcox, O. Ghattas, Non-linear model reduction for uncertainty quantification in large scale inverse problems, International Journal for Numerical Methods in Engineering 81(12) (2010) 15811608.

[60] C. Lieberman, K. Willcox, O. Ghattas, Parameter and state model reduction for large scale statistical inverse problems, SIAM Journal on Scientific Computing 32(5) (2010) 2523-2542.

[61] A. Nouy, C. Soize, Random field representations for stochastic elliptic boundary value problems and statistical inverse problems, European Journal of Applied Mathematics 25 (3) (2014) 339-373. doi:10.1017/ S0956792514000072

[62] T. Cui, Y. Marzouk, K. Willcox, Data-driven model reduction for the bayesian solution of inverse problems, International Journal for Numerical Methods in Engineering 102(5) (2015) 966-990.

[63] C. Soize, Random vectors and random fields in high dimension: parametric model-based representation, identification from data, and inverse problems, in: R. Ghanem, D. Higdon, H. Owhadi (Eds.), Handbook of Uncertainty Quantification, Vol. 2, Springer, Cham, Switzerland, 2017, Ch. 26, pp. 883-936. doi:10.1007/ 978-3-319-11259-6_30-1

[64] C. Soize, A nonparametric model of random uncertainties for reduced matrix models in structural dynamics, Probabilist Engineering Mechanics 15(3) (2000) 277-294. doi:10.1016/S0266-8920(99)00028-4

[65] C. Shannon, A mathematical theory of communication, The Bell system technical journal 27 (3) (1948) 379-423.

[66] E. Jaynes, Information theory and statistical mechanics, Physical review 106 (4) (1957) 620.

[67] M. Mignolet, C. Soize, Nonparametric stochastic modeling of linear systems with prescribed variance of several natural frequencies, Probabilistic Engineering Mechanics 23(2-3) (2008) 267-278. doi:10.1016/j. probengmech.2007.12.027

[68] C. Soize, Random matrix models and nonparametric method for uncertainty quantification, in: R. Ghanem, D. Hig don, H. Owhadi (Eds.), Handbook of Uncertainty Quantification, Vol. 1, Springer, Cham, Switzerland, 2017, pp 219-287. doi:10.1007/978-3-319-11259-6_5-1

[69] C. Chen, D. Duhamel, C. Soize, Probabilistic approach for model and data uncertainties and its experimental identification in structural dynamics: Case of composite sandwich panels, Journal of Sound and Vibration 294(1-2) (2006) 64-81. doi:10.1016/j.jsv.2005.10.013

[70] R. Capillon, C. Desceliers, C. Soize, Uncertainty quantification in computational linear structural dynamics for viscoelastic composite structures, Computer Methods in Applied Mechanics and Engineering 305 (2016) 154-172 doi:10.1016/j.cma.2016.03.012

[71] C. Soize, H. Chebli, Random uncertainties model in dynamic substructuring using a nonparametric probabilistic model, Journal of Engineering Mechanics ASCE 129(4) (2003) 449-457. doi:10.1061/(ASCE) 0733-9399(2003) 129:4(449)

[72] M. Mignolet, C. Soize, J. Avalos, Nonparametric stochastic modeling of structures with uncertain boundary conditions / coupling between substructures, AIAA Journal 51(6) (2013) 1296-1308. doi:10.2514/1. J051555

[73] M. Arnst, D. Clouteau, H. Chebli, R. Othman, G. Degrande, A non-parametric probabilistic model for groundborne vibrations in buildings, Probabilistic Engineering Mechanics 21(1) (2006) 18-34. 
[74] R. Ohayon, C. Soize, Advanced Computational Vibroacoustics - Reduced-Order Models and Uncertainty Quantification, Cambridge University Press, New York, 2014.

[75] E. Capiez-Lernout, C. Soize, Robust design optimization in computational mechanics, Journal of Applied Mechanics 75 (2) (2008) 021001. doi:10.1115/1.2775493

[76] M. Arnst, C. Soize, Identification and sampling of bayesian posteriors of high-dimensional symmetric positivedefinite matrices for data-driven updating of computational models, Computer Methods in Applied Mechanics and Engineering 352 (2019) 300-323. doi:10.1016/j.cma.2019.04.025

[77] M. Mignolet, C. Soize, Stochastic reduced order models for uncertain geometrically nonlinear dynamical systems, Computer Methods in Applied Mechanics and Engineering 197(45-48) (2008) 3951-3963. doi:10.1016/j . cma. 2008.03 .032

[78] E. Capiez-Lernout, C. Soize, M. MP., Post-buckling nonlinear static and dynamical analyses of uncertain cylindrica shells and experimental validation, Computer Methods in Applied Mechanics and Engineering 271(1) (2014) 210230 .

[79] C. Soize, C. Farhat, A nonparametric probabilistic approach for quantifying uncertainties in low-dimensional and high-dimensional nonlinear models, International Journal for Numerical Methods in Engineering 109 (6) (2017) 837-888. doi:10.1002/nme.5312

[80] O. Ezvan, A. Batou, C. Soize, Multilevel reduced-order computational model in structural dynamics for the low-and medium-frequency ranges, Computers \& Structures 160 (2015) 111-125. doi:10.1016/j.compstruc.2015. 08.007

[81] O. Ezvan, A. Batou, C. Soize, L. Gagliardini, Multilevel model reduction for uncertainty quantification in computational structural dynamics, Computational Mechanics 59 (2) (2017) 219-246. doi:10.1007/ s00466-016-1348-1

[82] R. Ohayon, C. Soize, Structural Acoustics and Vibration, Academic Press, San Diego, 1998. 